\title{
Cross-Layer Scheduling for OFDMA-based Cognitive Radio Systems with Delay and Security Constraints
}

\author{
Xingzheng Zhu, Bo Yang, Cailian Chen, Liang Xue, Xinping Guan, Fan Wu
}

\begin{abstract}
This paper considers the resource allocation problem in an Orthogonal Frequency Division Multiple Access (OFDMA) based cognitive radio (CR) network, where the CR base station adopts full overlay scheme to transmit both private and open information to multiple users with average delay and power constraints. A stochastic optimization problem is formulated to develop flow control and radio resource allocation in order to maximize the long-term system throughput of open and private information in CR system and ensure the stability of primary system. The corresponding optimal condition for employing full overlay is derived in the context of concurrent transmission of open and private information. An online resource allocation scheme is designed to adapt the transmission of open and private information based on monitoring the status of primary system as well as the channel and queue states in the CR network. The scheme is proven to be asymptotically optimal in solving the stochastic optimization problem without knowing any statistical information. Simulations are provided to verify the analytical results and efficiency of the scheme.
\end{abstract}

Index Terms-Cognitive radio, physical-layer security, delayaware network, full overlay, cross-layer scheduling.

\section{INTRODUCTION}

$\mathbf{T}$ HE emergency of high-speed wireless applications and increasing scarcity of available spectrum remind researchers of spectrum utilizing efficiency. The concept of $\mathrm{CR}$ provides the potential technology in increasing spectrum utilizing efficiency [1], [2] because CR allows unlicensed users (also known as secondary users (SUs)) to access some spectrum which is already allocated to primary user (PU) or

Copyright (c) 2013 IEEE. Personal use of this material is permitted. However, permission to use this material for any other purposes must be obtained from the IEEE by sending a request to pubs-permissions @ ieee.org.

The work was partially supported by National Basic Research Program of China under the grant no. 2010CB731803; by NSF of China under 61174127 61221003, 61290322, 61273181, 61374107, 61304131, U1405251; by Ministry of Education of China under NCET-13-0358; by the Research Found for the Doctoral Program of Higher Education under Grants 20110073120025 and 20110073130005; by the Shanghai Municipal Science and Technology Commission, China under 14511107903 and 13QA1401900; by Huawei Innovation Research Program with no. YB2014030021; by the Scientific Research Project of Hebei Education Department BJ2014019;

X. Zhu, B. Yang, C. Chen and X. Guan are with Department of Automation, Shanghai Jiao Tong University, and Key Laboratory of System Control and Information Processing, Ministry of Education of China, Shanghai 200240, China (Emails: \{wendyzhu, bo.yang, cailianchen, xpguan\}@ sjtu.edu.cn). B. Yang, C. Chen and X. Guan are also with the Cyber Joint Innovation Center, Hangzhou, China. L. Xue is with School of Information and Electrical Engineering, Hebei University of Engineering, Handan 056038, China (Email: liangxue@hebeu.edu.cn). F. Wu is with the Department of Computer Science and Engineering, Shanghai Key Laboratory of Scalable Computing and Systems, Shanghai Jiao Tong University, Shanghai 200240, China (E-mail: fwu@cs.sjtu.edu.cn). B. Yang is the corresponding author of this paper. licensed user who has the authority to access the spectrum by spectrum sensing [3], [4]. As another promising technology of high speed wireless communication system, OFDMA is a candidate for CR systems [1] due to its flexibility in allocating spectrum among SUs [5]. Hence, OFDMA-based CR networks are catching great attention [6], [7]. This paper focuses on an OFDMA-based CR network without loss of generality.

In order to exploit the capacity of the whole OFDMAbsed CR system, this paper aims at maximizing the secondary network capacity in consideration of the whole system transmission efficiency. Thus, the following three main issues should be considered.

Firstly, an efficient spectrum sharing scheme is essential for exploiting the unused spectrum in OFDMA-based CR network. When a SU wants to access some spectrum, it must ensure that the spectrum is not accessed by any PU or adapt its parameter to limit the interference to PU. Both of these two mentioned spectrum utilization manners, known as overlay and underlay schemes, are conservative in some ways, since they ignore the PU's ability to tolerate some inference.

Secondly, due to that CR networks as well as many other kinds of wireless communication systems have a nature of broadcast, security issues at physical layer have always been unavoidable in designing CR systems. Furthermore, to SUs, it is obviously practical that there exist both private and open transmission requirements. Then, the scheduling among these two different kinds of transmission should be considered. In addtion, delay performance is an indispensable quality of service (QoS) index in scheduling different transmissions.

Last but not least, the dynamic nature of OFDMA-based $\mathrm{CR}$ communication system brings another big challenge. The random arrival of user requests (from both $\mathrm{PU}$ and SU) and time-varying channel states renders dynamic resource allocation instead of fixed ones in exploiting the OFDMA secondary network capacity.

Aiming at the above issues, the contributions of this paper are threehold:

- First, this paper adopts a novel full overlay spectrum accessing scheme by exploiting PU's tolerance to interference. Besides, the theoretic proof of full overlay's optimality is given in the presence of both open and private transmissions.

- Second, a joint encoding model is introduced to allow both private and open transmissions towards SUs with the full overlay spectrum sharing scheme. A dynamic resource allocation scheme consisting of flow control 
and radio resource allocation is developed by solving a formulated stochastic optimization problem under the delay and power constraints.

- Third, the proposed dynamic resource allocation scheme is proven to be close to optimality although its implementation only depending on instantaneous information.

This paper is organized as follows. Section II presents the related work. In Section III, we introduce the system model and relevant constraints in detail. Section IV formulates the problem. In Section V, we introduce our cross-layer optimization algorithm. We give the performance bound and stability results in Section VI. Two different implementations are proposed in Section VII. In Section VIII, some simulation results are shown. Finally, we conclude this paper in Section IX.

\section{RELATED WORK}

There have been many works on spectrum sharing in OFDMA-based CR networks [8]-[10]. According to [11], [12], the access technology of the SUs can be divided in two categories: spectrum underlay and spectrum overlay. The first category means that SUs can access licensed spectrum during PUs' transmission, while as is mentioned in [12], this approach imposes severe constraints on the transmission power of SUs such that they can operate below the noise floor of PUs, e.g, in [8], [13], [14]. The second category means that SUs can only access licensed spectrum when the PU is idle, e.g, in [9], [10], [15]-[17]. Considering both these two strategies suffer from some drawbacks, the authors in [18] propose a new cognitive overlay scheme requiring SUs to assess and control their interference impacts on PUs. In general, the cognitive base station (CBS) controls the aggregate interference to primary transmission by allowing SUs to monitor channel quality indicators (CQIs), power-control notifications and ACK/NAK of primary transmission. In this paper, this novel thought is extended into an OFDMA-based CR system.

On the other hand, dynamic resource allocation plays a critical role in exploiting OFDMA network capacity. The overall performance as well as the multiuser diversity of the system can be improved by proper dynamic resource allocation [17], [19]-[25]. Thus, dynamic resource allocation in OFDMAbased CR system has been attracting more attention recently. The corresponding spectrum sharing schemes in [8]-[10] are all realized by dynamic resource allocation.

Besides the interference constraints, the works of delay aware transmission are also quite relative to this paper. Huang and Fang in [26] investigate both reliability and delay constraints in routing design for wireless sensor network. Cui et al. in [27] summarize three approaches to deal with delayaware resource allocation in wireless networks. A constrained predictive control strategy is proposed in [28] to compensate for network-induced delays with stability guarantee. Those three methods are based on large deviation theory, Markov decision theory and Lyapunov optimization techniques. As to the first two methods, they have to know some statistical information on channel state and random arrival data rate to design algorithm, while these prior knowledge is expensive to get, even unavailable. To overcome this problem, many authors pay attention to Lyapunov optimization techniques. References [29] and [30] investigate scheduling in multihop wireless networks and resource allocation in cooperative communications, respectively as two typical applications of Lyapunov optimization in delay-limited system. In this paper, we utilize this tool to dispose the resource allocation problem in OFDMA-based CR networks.

As for secure transmission, Shannon's information theory laid the foundation for information-theoretic security [31] and the concept of wire-tap channel was proposed in [32]. There has been some research on exploiting security capacity in OFDMA network by dynamic resource allocation, such as in [33] and [34]. In CR area, the study of secure transmission from information-theoretic aspect is very limited. Pei et al. in [35] first investigate secrecy capacity of the secure multipleinput single-output (MISO) CR channel. Kwon et al. in [36] utilize the concept of security capacity to explore MISO CR systems where the secondary system secures the primary communication in return for permission to use the spectrum. Both these two works focus on only private message transmission. The security and common capacity of cognitive interference channels is analyzed in [37]. The entire capacity of a MIMO broadcast channel with common and confidential messages is obtained in [38]. The paper [39] considers the problem of optimizing the security and common capacity of an OFDMA downlink system by dynamic resource allocation. This paper further considers the transmissions of private and open flows in CR networks with delay constraints.

\section{System Model}

The system model consists of multiple primary links and multiple secondary links as Fig. 1 shows. The total bandwidth $B$ is divided into $M$ subcarriers equally using Orthogonal Frequency Division Multiplexing (OFDM). Assume that $M=B$ holds for simplicity of expression. The subcarrier set of the network is denoted as $\mathbf{M}=\{1,2, \cdots, M\}$ and $m \in \mathbf{M}$ denotes subcarrier index. The downlink case is considered. The primary link is from a single primary base station (PBS) to $K$ PUs. Secondary links are from a common CBS to $N$ SUs. We denote $k \in\{1,2, \cdots, K\}$ and $n \in\{1,2, \cdots, N\}$ as the indexes of PU and SU respectively. The system operates in slotted time, and $T$ is the length of a time slot. Hereafter, $[t T,(t+1) T)$ is just denoted by $t$ for brevity.

The set of subcarriers occupied by PU $k$ on timeslot $t$ is denoted as $\boldsymbol{\Gamma}_{k}^{P U}(t)=\left\{\tau_{1}^{k}(t), \tau_{2}^{k}(t), \cdots, \tau_{m^{k}(t)}^{k}(t)\right\}$ where $m^{k}(t)$ is the number of subcarriers occupied by $\mathrm{PU} k$ and $\boldsymbol{\Gamma}_{k}^{P U}(t) \subseteq\{1,2, \cdots, M\}$. The power set $\mathbf{P}_{k}^{P U}(t)=$ $\left\{P_{k}^{m}(t) \mid m \in \mathbf{M}\right\}$ is the set of transmission power from PBS to PU $k$, where for $m \in \boldsymbol{\Gamma}_{k}^{P U}(t), P_{k}^{m}(t)>0$, else $P_{k}^{m}(t)=0$. For brevity, we will omit the time index $(t)$ somewhere in further discussion. $\boldsymbol{P}^{S U}=\left\{p_{n}^{m} \mid \forall n, \forall m\right\}$ denotes the overall SUs power allocation policy set and $p_{n}^{m}$ represents the power allocated by CBS to user $n$ in subcarrier $m$. Denote $\Gamma_{n}^{S U}=\left\{\varpi_{n}^{m} \mid \forall m\right\}$ as the subcarrier assignment policy of SU $n$, where $\varpi_{n}^{m}$ is either 1 representing subcarrier $m$ is assigned to $\mathrm{SU} n$, or 0 otherwise. Then let $\boldsymbol{\Gamma}^{S U}=\left\{\boldsymbol{\Gamma}_{n}^{S U}(t), \forall n\right\}$ be the overall subcarrier assignment policy of secondary network. 
Due to the orthogonal properties of OFDMA technology, there exists no mutual influence between every two SUs. However, there exists mutual interference between the primary and secondary networks when PU and SU access in the same subcarrier.

The channel gains include the one of secondary user $n$ on subcarrier $m, h_{n}^{m}$ and the one of primary user $k$ on subcarrier $m, H_{k}^{m}$. The additive white gaussian noise (AWGN) is $\sigma^{2}$. The corresponding subcarrier gain-to-noiseratio $^{1}(\mathrm{C} / \mathrm{I})$ in slot $t$ are thus defined as $a_{n}^{m}(t)=\frac{h_{n}^{m}(t)^{2}}{\sigma^{2}}$ and $A_{k}^{m}=\frac{H_{k}^{m}(t)^{2}}{\sigma^{2}}$ respectively as illustrated in Fig.1. The set $\boldsymbol{a}(t)=\left\{A_{k}^{m}(t), a_{n}^{m}(t), \forall n, \forall m, \forall k\right\}$ represents the system channel state information (CSI). All channels are assumed to be slow fading, and thus $\boldsymbol{a}(t)$ remains fixed during one slot and changes between two [40]. In this work, there exists an reasonable assumption that the system CSI is known to BS. As in [41], BS can get full-CSI by utilizing pilot symbols and CSI feedback process. Besides, at the beginning of every slot, PU reports $P_{k}^{m} A_{k}^{m}$ to PBS. For example, the PU reports a received-signal-strength index to PBS in packets such as RSSI reports. We assume the CBS will listen to the information to derive $P_{k}^{m} A_{k}^{m}$ before accessing subcarrier $m$ [18], [41].

Denote $h_{k S}^{m}$ as the cross-link interference channel gain from CBS to PU $k$ on subcarrier $m$ and let $a_{k S}^{m}=\frac{h_{k S}^{m}{ }^{2}}{\sigma^{2}}$. Similarly, denote $h_{n P}^{m}$ as the cross-link interference channel gain from PBS to SU $n$ on subcarrier $m$ and let $a_{n P}^{m}=\frac{h_{n P}^{m}{ }^{2}}{\sigma^{2}}$. It is assumed that $a_{k S}^{m}$ and $a_{n P}^{m}$ can be got by the CBS. $a_{k S}^{m}$ can be estimated by CBS from the PU feedback signal based on reciprocity. $a_{n P}^{m}$ can be estimated by SUs through training and sensing and the estimation results are sent to CBS [41]. Beyond that, information about cross-link channel state could also be measured periodically by a band manager either [8], [42].

Compared to pervious work, this paper considers a more complicated and practical situation of SU transmission. The CBS transmits both private and open data to each SU as Fig.2 shows. The private data has security requirement and open data has long-term time-average delay constraint. Instead of that both open and private data have delay constraint, only delay constraints on open transmissions are considered in this paper for simplifying the mathematic expressions, since the handling of delay constraint in secure transmission is totally the same as open transmission. Actually, in real wireless communication systems, there exists some private transmission having no strict delay constraint, e,g. updating contact information in mobile devices. At the beginning of every time slot, random data packets arrive at CBS. CBS decides whether to admit it into the system or not. Besides, CBS is also in charge of resource allocation to assign power and subcarriers among SUs. CBS utilizes the information of data queue and CSI to allocate resources. The system performance can be optimized and the queuing delay of open data can be ensured to fulfill by flow control and resource allocation.

In the side of CBS, the amount of open data packet of SU $n, D_{n}^{o}(t)$, and private data, $D_{n}^{p}(t)$ that arrive at CBS during

\footnotetext{
${ }^{1}$ Also called gain-to-noise-plus-interference-ratio when SU and PU access in the same subcarrier.
}

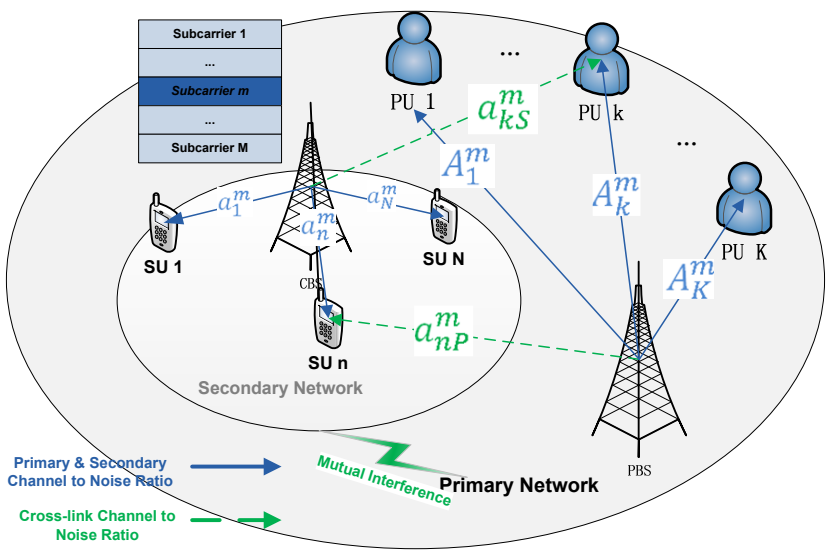

Fig. 1. General network model

slot $t$ are independent identically distributed (i.i.d) stochastic processes, e,g. Bernoulli processes, with the long-term average arrival rates $\lambda_{n}^{o}$ and $\lambda_{n}^{p}$, and their upper bounds are $\mu_{\max }$ and $D_{\max }$, respectively. These packets can not be transmitted to target users instantaneously due to the time-varying channel conditions and they are enqueued at the CBS. However, only parts of these packets are admitted into each queue towards each user for stability reason to be specified later. The amounts of open and private data admitted by respect queues are $T_{n}^{o}(t)$ and $T_{n}^{p}(t)$ and CBS is in charge of determining $T_{n}^{o}(t)$ and $T_{n}^{p}(t)$ according to a certain principle which would be specified in Section V.

\section{A. Capacity model}

In OFDMA-based CR networks, SU and PU can access in the same subcarrier with mutual interference. However, due to the characteristic of OFDMA networks, each subcarrier can not be assigned to more than solitary user in any secondary or primary network. Thus the following formulation is set to ensure the limitation in CBS:

$$
0 \leq \sum_{n=1}^{N} \varpi_{n}^{m} \leq 1, \quad \forall m
$$

CBS will realize the occupied subcarrier set $\Gamma_{k}^{P U}=$ $\left\{m \mid P_{k}^{m}>0, \forall m\right\}$, and we denote $\boldsymbol{\Gamma}_{S U}=\{1,2, \cdots, M\}-$ $\bigcup_{k=1}^{K} \Gamma_{k}^{P U}$. Thus the transmission rates of PU and SUs can be analysed by dividing $M$ subcarriers into two parts: one is $m \in \bigcup_{k=1}^{K} \boldsymbol{\Gamma}_{k}^{P U}$ where there exists interference between PU and SUs; another is $m \in \Gamma_{S U}$ which means SUs can access these subcarriers without influencing primary link. Thus according to information theory the transmission rate of PU $k$ on subcarrier $m$ is:

$$
R_{k}^{m}= \begin{cases}\log _{2}\left(1+\frac{P_{k}^{m} A_{k}^{m}}{1+a_{k S}^{m} p_{n^{\prime}}^{m}}\right) & m \in \boldsymbol{\Gamma}_{k}^{P U}, n^{\prime} \in \tilde{\boldsymbol{\Gamma}}_{m} \\ 0 & m \in \boldsymbol{\Gamma}_{S U}\end{cases}
$$


where $\tilde{\boldsymbol{\Gamma}}_{m}$ is the set of SUs accessing subcarrier $m$. Furthermore, since in secondary network, only one SU can access one subcarrier, $n^{\prime}$ is the only one element in set $\tilde{\boldsymbol{\Gamma}}_{m}$.

It should be noticed that the total transmission rate in an OFDMA network equals to the sum rates on all subcarriers. So the transmission rate of $\mathrm{PU}$ is:

$$
R_{k}^{P U}=\sum_{m \in \boldsymbol{\Gamma}_{k}^{P U}} R_{k}^{m}
$$

The channel capacities of SU $n$ on subcarrier $m$ can be expressed as:

$$
C_{n}^{m}= \begin{cases}\log _{2}\left(1+\frac{p_{n}^{m} a_{n}^{m}}{1+P_{k^{\prime}}^{m} a_{n P}^{m}}\right) & m \in \bigcup_{k=1}^{K} \boldsymbol{\Gamma}_{k}^{P U}, k^{\prime} \in \hat{\boldsymbol{\Gamma}}_{m} \\ \log _{2}\left(1+p_{n}^{m} a_{n}^{m}\right) & m \in \boldsymbol{\Gamma}_{S U}\end{cases}
$$

where $\hat{\boldsymbol{\Gamma}}_{m}$ is the set of PUs accessing subcarrier $m$. Furthermore, since only one PU can access one subcarrier, $k^{\prime}$ is the only one element in set $\hat{\boldsymbol{\Gamma}}_{m}$. Denote $R_{n}^{S U}=\sum_{m} C_{n}^{m}$ as the sum transmission rate of $\mathrm{SU} n$ without consideration of security.

By introducing the joint transmission model, open and private data of one SU can be transmitted simultaneously. Open message is jointly encoded with security message as random codes. In this way, although open message may be decoded by eavesdroppers, security message would be perfectly secure if the channel fading is properly utilized [43]. According to the theory of physical-layer security [34], if the transmission rate of private data is less than security capacity, the proposed joint-encoding model can at least realize physical-layer security in theory. [44], [45] propose physical-layer security realization applications using error correcting codes and preprocessor, which lays the foundation of realizing physicallayer security of the joint encoding model. For each SU, CBS makes decision if his secure data could be transmitted in this slot and this decision is expressed as the secure transmission control vector $\zeta=\left(\zeta_{1}, \zeta_{2}, \cdots, \zeta_{N}\right)$. The indicator variable $\zeta_{n}=1$ implies that private and open messages are encoded at rate $\hat{R}_{n}^{p}$ and $R_{n}^{S U}-\hat{R}_{n}^{p}$ respectively in timeslot $t$ and $\zeta_{n}=0$ means that only open messages can be transmitted at rate $R_{n}^{S U}$.

When CBS is transmitting private messages to $\mathrm{SU} n$, all the other SUs except SU $n$ are treated as potential eavesdroppers [34]. According to [46], subject to perfect private of SU n, the instantaneous private rate of SU $n$ on subcarrier $m$ is the achievable channel capacity minus the highest eavesdropper capacity if there is no cooperation among eavesdroppers. For each SU $n$, we define the most potential eavesdropper on subcarrier $m$ as SU $\tilde{n}$ and $\tilde{n}=\underset{n^{\prime} n^{\prime} \neq n}{\arg \max } a_{n^{\prime}}^{m}$. So the security capacity of SU $n$ on subcarrier $m$ is:

$\hat{R}_{n}^{m p}= \begin{cases}{\left[C_{n}^{m}-\log _{2}\left(1+\frac{p_{n}^{m} b_{n}^{m}}{1+P_{k^{\prime}}^{m} b_{n P}^{m}}\right)\right]^{+}} & m \in \boldsymbol{\Gamma}_{k}^{P U}, k^{\prime} \in \hat{\boldsymbol{\Gamma}}_{m} \\ {\left[C_{n}^{m}-\log _{2}\left(1+p_{n}^{m} b_{n}^{m}\right)\right]^{+}} & m \in \boldsymbol{\Gamma}_{S U}\end{cases}$

where $[\cdot]^{+}=\max \{\cdot, 0\}, b_{n}^{m}=a_{\tilde{n}}^{m}$ and $b_{n P}^{m}$ is the cross-link CSI from PBS to SU $\tilde{n}$ on subcarrier $m$. Obviously, $\hat{R}_{n}^{p}=$ $\sum_{m \in \mathrm{M}} \hat{R}_{n}^{m p}$. Thus the achievable private rate of user $n$ is:

$$
R_{n}^{p}=\zeta_{n} \hat{R}_{n}^{p}
$$

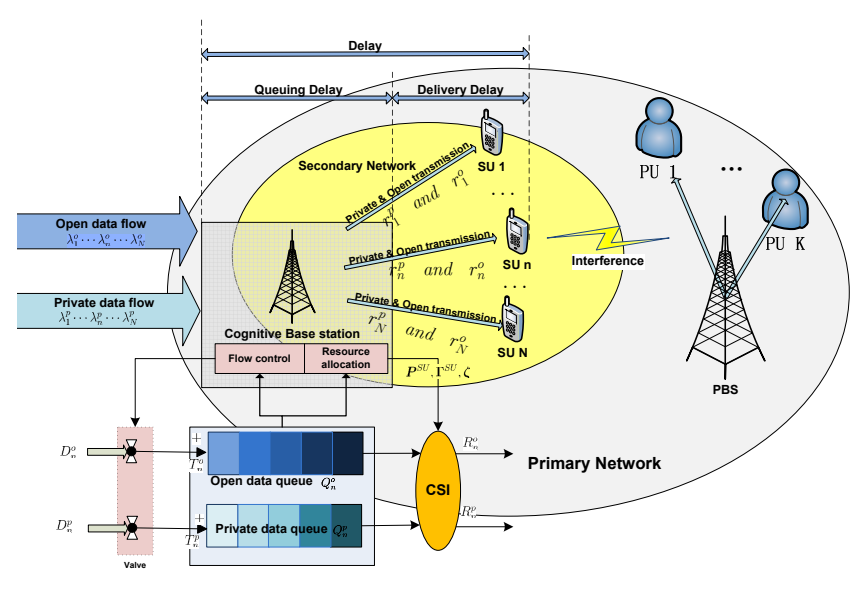

Fig. 2. Transmission model of secondary network

and the open rate of user $n$ is: $R_{n}^{o}=R_{n}^{S U}-R_{n}^{p}$.

\section{B. Queuing model}

There exist data queues in both PBS and CBS. Although we want to maximize the weighted throughput of SUs, PU queue stability is a constraint in ensuring that PU's longterm throughput is not affected by SU's transmission. It is assumed that the transmission rate of PBS without interference is sufficient to serve PU's demand. However, the primary network and the secondary network will be influenced by each other if they work on the same channel. The transmission rate decrease of $\mathrm{PU}$ is due to the interference brought by SU transmission, while the CBS can adjust its schedule to limit interference in order to ensure that PU's time-varying rate demands can be satisfied. Later, the notation of queue stability will be used to measure whether PU's demand can be fulfilled. In [18], the interference is limited by that PU queue is kept stable under the influence caused by the only one SU access. We continue to utilize this technique in scheduling our multi-SU access system.

First, it is necessary to introduce the concept of strong stability. As a discrete time process, $Q(t+1)=[Q(t)-$ $S(t)]^{+}+D(t)$ is strongly stable if:

$$
\limsup _{t \rightarrow \infty} \frac{1}{t} \sum_{\tau=0}^{t-1} \mathbb{E}\{Q(\tau)\}<\infty
$$

In particular, a multi-queue network is stable when all queues of the network are strongly stable. According to Strong Stability Theorem in [47], for finite variable $S(t)$ and $D(t)$, strong stability implies rate stability of $Q(t)$. The definition of rate stability can be found in [47] and omitted here.

Furthermore, according to Rate Stability Theorem in [47], $Q(t)$ is rate stable if and only if $d \leq s$ holds where $d=$ $\lim _{t \rightarrow \infty} \frac{1}{t} \sum_{\tau=0}^{t-1} D(\tau)$ and $s=\lim _{t \rightarrow \infty} \frac{1}{t} \sum_{\tau=0}^{\overline{t-1}} S(\tau)$.

Since the data can not be delivered instantly to PUs or SUs, there are data backlogs in the PBS and CBS waiting for transmitting to respective users. 
1) PU queue: In PBS, the data queue of PU $k$ is updated as following:

$$
Q_{k}(t+1)=\left[Q_{k}(t)-R_{k}^{P U}(t)\right]^{+}+D_{k}^{P U}(t)
$$

where $D_{k}^{P U}(t)$ is the amount of data packets randomly arriving at PBS during slot $t$ with the destination of PU $k$. We assume $D_{k}^{P U}(t)$ is an i.i.d stochastic process with its upper bound of $D_{\max }^{P U}$ and its long-term average arrival rates $\lambda_{k}=$ $\lim _{t \rightarrow \infty} \frac{1}{t} \sum_{\tau=0}^{t-1} D_{k}^{P U}(\tau)$. As it has been mentioned before, $Q_{k}$ should be kept stable by limiting SUs' interference to primary link. As Rate Stability Theorem shows, $Q_{k}$ is rate stable if and only if $r_{k}^{P U} \geq \lambda_{k}$ where $r_{k}^{P U} \triangleq \lim _{t \rightarrow \infty} \frac{1}{t} \sum_{\tau=0}^{t-1} R_{k}^{P U}(\tau)$. Therefore, if $\mathrm{PU}$ system is strongly stable, its long-term transmission is not affected by SUs.

2) SU data queues: In CBS, there exist actual data queues of open and private data which are represented by $Q_{n}^{o}$ and $Q_{n}^{p}$ respectively for all $n \in\{1, \cdots, N\}$. These queues are updated as follows:

$$
\begin{aligned}
& Q_{n}^{o}(t+1)=\left[Q_{n}^{o}(t)-R_{n}^{o}(t)\right]^{+}+T_{n}^{o}(t) \\
& Q_{n}^{p}(t+1)=\left[Q_{n}^{p}(t)-R_{n}^{p}(t)\right]^{+}+T_{n}^{p}(t)
\end{aligned}
$$

All $Q_{k}, Q_{n}^{o}$ and $Q_{n}^{p}$ have initial values of zero. We define $t_{n}^{o} \triangleq \lim _{t \rightarrow \infty} \frac{1}{t} \sum_{\tau=0}^{t-1} T_{n}^{o}(\tau), t_{n}^{p} \triangleq \lim _{t \rightarrow \infty} \frac{1}{t} \sum_{\tau=0}^{t-1} T_{n}^{p}(\tau)$ as the long-term time-average admission rates of open data and private data respectively. The long-term time-average service rates of $Q_{n}^{o}$ and $Q_{n}^{p}$ are also defined as: $r_{n}^{o} \triangleq$ $\lim _{t \rightarrow \infty} \frac{1}{t} \sum_{\tau=0}^{t-1} R_{n}^{o}(\tau)$ and $r_{n}^{p} \triangleq \lim _{t \rightarrow \infty} \frac{1}{t} \sum_{\tau=0}^{t-1} R_{n}^{p}(\tau) . Q_{n}^{o}$ and $\stackrel{t \rightarrow \infty}{Q_{n}^{p}}$ should be kept strongly stable in order to ensure the rate requirements of open and private date can be supported by the CR system, which means $t_{n}^{o} \leq r_{n}^{o}$ and $t_{n}^{p} \leq r_{n}^{p}$ hold.

Virtual queues of open data, $X_{n}^{o}(t)$, and private data $X_{n}^{p}(t)$ are introduced in (8) and (9) to assist in developing our algorithms, which would guarantee that the actual queues $Q_{n}^{o}$ and $Q_{n}^{p}$ are bounded deterministically in the worst case.

$$
\begin{aligned}
& X_{n}^{o}(t+1)=\left[X_{n}^{o}(t)-T_{n}^{o}(t)\right]^{+}+\mu_{n}^{o}(t) \\
& X_{n}^{p}(t+1)=\left[X_{n}^{p}(t)-T_{n}^{o}(t)\right]^{+}+\mu_{n}^{p}(t)
\end{aligned}
$$

Denote $\mu_{n}^{o}$ and $\mu_{n}^{p}$ as the virtual admission rates of open data and private data, which are upper bounded by $D_{n}^{o}$ and $D_{n}^{p}$ respectively. Notice that $X_{n}^{o}, X_{n}^{p}, \mu_{n}^{o}$ and $\mu_{n}^{p}$ do not stand for any actual queue and data. They are only generated by the proposed algorithms. According to queuing theory, when $X_{n}^{o}$ and $X_{n}^{p}$ are stable, the long-term time-average value of $\mu_{n}^{o}$ and $\mu_{n}^{p}$ would satisfy:

$$
\begin{aligned}
& \nu_{n}^{o}=\lim _{t \rightarrow \infty} \frac{1}{t} \sum_{\tau=0}^{t-1} \mu_{n}^{o}(\tau) \leq t_{n}^{o} \\
& \nu_{n}^{p}=\lim _{t \rightarrow \infty} \frac{1}{t} \sum_{\tau=0}^{t-1} \mu_{n}^{p}(\tau) \leq t_{n}^{p}
\end{aligned}
$$

To summarise, as shown in Fig. 2 the control space $\chi$ of the system can be expressed as $\chi=\left\{\mathbf{P}^{S U}, \boldsymbol{\Gamma}^{S U}, \zeta, \mathbf{T}\right\}$, which includes admission control $\mathbf{T}=\left\{T_{n}^{o}, T_{n}^{p} \mid \forall n\right\}$, power control decision $\mathbf{P}^{S U}$, subcarrier assignment $\boldsymbol{\Gamma}^{S U}$ and security transmission control $\zeta$.

\section{Basic constraints}

1) Power consumption constraint: Let $E \triangleq \sum_{\forall n, \forall m} p_{n}^{m}$ as total power consumption of the whole system in one time slot. There exists a physical peak power limitation $P_{\max }$ that $E$ cannot exceed at any time:

$$
0 \leq E \leq P_{\max }
$$

The long-term time-average power consumption also has an upper bound $P_{\text {avg }}$, which is proposed for energy conservation:

$$
e \leq P_{a v g}
$$

where $e=\lim _{t \rightarrow \infty} \frac{1}{t} \sum_{\tau=0}^{t-1} \mathbb{E}\{E(\tau)\}$

2) Delay-limited model: The queuing delay is defined as the time a packet waits in a queue until it can be transmitted. Each SU has a long-term time-average queuing delay $\rho_{n}^{o}$ for its open data transmission. To each SU, it proposes a delay constraint $\rho_{n}$ as in (14) for its open transmission.

$$
\rho_{n}^{o} \leq \rho_{n}
$$

\section{PRoblem Formulation}

Considering the simplicity and understandability of mathematic analysis, a special case of one single primary link is considered in the following. In the single PU case, the only one PU is indexed with number 0 . In part C of Section V, the general results of multi-PU case are listed for completeness.

\section{A. Optimization objective and constraints}

Following above descriptions, the objective of this paper is to improve throughput of secondary network while ensuring stability of primary network. So the problem is formulated as: Maximize the sum weighted admission rates of all SUs and stabilize the PU data queue $Q_{0}$ at the same time. Let $\theta_{n}$ and $\varphi_{n}$ for all $n$ be the nonnegative weights for private and open data throughput. Then the optimal problem can be formulated as:

$$
\begin{array}{cl}
\text { Maximize } & \sum_{n=1}^{N}\left\{\theta_{n} t_{n}^{p}+\varphi_{n} t_{n}^{o}\right\} \\
\text { Subject to: } & 0 \leq t_{n}^{p} \leq \lambda_{n}^{p}, \forall n \\
& 0 \leq t_{n}^{o} \leq \lambda_{n}^{o}, \forall n \\
& \mathbf{t}=\left(t_{n}^{p}, t_{n}^{o}\right) \in \mathbf{\Upsilon} \\
& \limsup _{t \rightarrow \infty} \frac{1}{t} \sum_{\tau=0}^{t-1} \mathbb{E}\left\{Q_{0}(\tau)\right\}<\infty
\end{array}
$$

(13), (14)

where $\Upsilon$ is the network capacity region of secondary links. Define the service rate vector as $v=\left(r_{n}^{o}, r_{n}^{p}\right)$. The definition of network capacity region $\Upsilon$ is the region of all non-negative service rate vectors $v$ for any possible control actions [47]. When the CBS takes a kind of control policy under a certain channel condition, the secondary links will have a decided network capacity and the network capacity region is the set of network capacities under all possible control policies and all channel conditions. In the proposed system, the control policy of CBS should fulfill subcarrier assignment rule (1), 
peak power constraint (12) and stabilize all queues including actual queues and virtual queues. So actually, the control policy that can achieve the network capacity region should satisfy the following constraints:

$$
\begin{aligned}
& (1),(12) \\
& \lim \sup _{t \rightarrow \infty} \frac{1}{t} \sum_{\tau=0}^{t-1} \mathbb{E}\left\{Q_{n}^{o}(\tau)\right\}<\infty \\
& \lim \sup _{t \rightarrow \infty} \frac{1}{t} \sum_{\tau=0}^{t-1} \mathbb{E}\left\{Q_{n}^{p}(\tau)\right\}<\infty \\
& \lim \sup _{t \rightarrow \infty} \frac{1}{t} \sum_{\tau=0}^{t-1} \mathbb{E}\left\{X_{n}^{o}(\tau)\right\}<\infty \\
& \lim \sup _{t \rightarrow \infty} \frac{1}{t} \sum_{\tau=0}^{t-1} \mathbb{E}\left\{X_{n}^{p}(\tau)\right\}<\infty
\end{aligned}
$$

Theoretically, we can get the optimal solution to (15) if we get the distribution of the system CSI and external data arrival rate beforehand. However, this information can not be obtained accurately. In this paper an online algorithm requiring only current information of queue state and channel state is proposed and will be described in detail then.

\section{B. Optimality of $S U$ overlay}

Before detailing the control algorithm, it should be specified the conditions that make SU overlay play a positive role in this cognitive transmission model other than traditional access methods. We focus on presenting a sufficient condition on overlay for constant channel conditions here, then we will extend it to time-varying situation.

In the case of static network condition, the optimal problem of SUs' weighted throughput is simplified as

$$
\begin{array}{cc}
\text { Maximize: } & \sum_{n=1}^{N}\left\{\theta_{n} r_{n}^{p}+\varphi_{n} r_{n}^{o}\right\} \\
\text { Subject to } & r_{0}^{P U}=\lambda_{0}
\end{array}
$$

where we only consider the optimal case when $r_{0}^{P U}=\lambda_{0}$. Notice here, the system maximal weighted sum data rate under full overlay scheme must be greater than or at least no worse than that when SU can only access the subcarrier which is not occupied by PU. It is easy to understand that full overlay is a more general access scheme than spectrum overlay which is a special access situation. We can get an intuition that when all subcarriers are assumed to be accessed by PU, SU data rate would be positive under full overlay scheme instead of zero under traditional overlay scheme. Thus what we want to prove is the sufficient condition of that SUs perform better in consideration of PU transmission other than accessing the licensed subcarrier roughly. Let $\kappa$ be the fraction of time that PU is actively transmitting, thus:

$$
\begin{aligned}
r_{0}^{P U} & =\kappa \sum_{m \in \boldsymbol{\Gamma}_{0}^{P U}} \log _{2}\left(1+\frac{A_{0}^{m} P_{0}^{m}}{1+a_{0 S}^{m} p_{n}^{m}}\right) \\
r_{n}^{p} & =\left\{\sum _ { m \in \boldsymbol { \Gamma } _ { 0 } ^ { P U } } \left\{(1-\kappa)\left[\log _{2}\left(1+a_{n}^{m} p_{n}^{m}\right)-\log _{2}\left(1+b_{n}^{m} p_{n}^{m}\right)\right]^{+}\right.\right. \\
& \left.+\kappa\left[\log _{2}\left(1+\frac{p_{n}^{m} a_{n}^{m}}{1+P_{0}^{m} a_{n P}^{m}}\right)-\log _{2}\left(1+\frac{p_{n}^{m} b_{n}^{m}}{1+P_{0}^{m} b_{n P}^{m}}\right)\right]^{+}\right\} \\
& \left.+\sum_{m \in \boldsymbol{\Gamma}_{S U}}\left[\log _{2}\left(1+a_{n}^{m} p_{n}^{m}\right)-\log _{2}\left(1+b_{n}^{m} p_{n}^{m}\right)\right]^{+}\right\} \zeta_{n} \\
r_{n}^{o} & =\sum_{m \in \boldsymbol{\Gamma}_{S U}} \log _{2}\left(1+a_{n}^{m} p_{n}^{m}\right)+\sum_{m \in \boldsymbol{\Gamma}_{0}^{P U}}\left[\kappa \log _{2}(1+\right.
\end{aligned}
$$

$$
\left.\left.\frac{p_{n}^{m} a_{n}^{m}}{1+P_{0}^{m} a_{n P}^{m}}\right)+(1-\kappa) \log _{2}\left(1+a_{n}^{m} p_{n}^{m}\right)\right]-r_{n}^{p}
$$

We have the following lemma:

Lemma 1: In high SINR region, a sufficient condition for full overlay to be optimum in SU $n$ accessing subcarrier $m$ (both security and open transmission) is:

$$
a_{0 S}^{m} \leq \min \left\{C_{n m}^{1}, C_{n m}^{2}\right\}, \forall m
$$

where $C_{n m}^{1}=b_{n}^{m} /\left[\left(1+P_{0}^{m} b_{n P}^{m}+b_{n}^{m} P_{\max }\right) \log _{2}(1+\right.$ $\left.\left.b_{n}^{m} P_{\max }\right)\right], C_{n m}^{2}=a_{n}^{m} /\left[\left(1+P_{0}^{m} a_{n P}^{m}+a_{n}^{m} P_{\max }\right) \log _{2}(1+\right.$ $\left.\left.a_{n}^{m} P_{\max }\right)\right]$.

We can have an intuitive explanation on Lemma 1, for SU $n$ 's accessing subcarrier $m$. If the cross link (from CBS to primary link) condition is bad enough (worse than weighted CBS-to-SU channel condition $C_{n m}^{2}$ and weighted CBS-toeavesdropper channel condition $C_{n m}^{1}$ ), the full overlay scheme would be the optimal scheme when both security and open transmission happen. The proof of Lemma 1 can be found in Appendix C of reference [48] and omitted here for simplicity.

It would be obvious to derive the following lemma on sufficient condition of optimality of the whole system overlay. Thus we get:

Lemma 2: In high SINR region, a sufficient condition for full overlay to be optimum in the whole OFDMA-based CR system is:

$$
a_{0 S}^{m} \leq \min _{n}\left\{C_{n m}^{1}, C_{n m}^{2}\right\}, \forall m
$$

Notice that, the sufficient condition does not mean that subcarrier $m \in \boldsymbol{\Gamma}_{0}^{P U}$ would provide a greater data rate than $m^{\prime} \in \boldsymbol{\Gamma}_{S U}$ under the same power allocation scheme. It means that for $m \in \Gamma_{0}^{P U}$, full overlay would achieve the optimal result other than any other access policy such as partial overlay or underlay. We assume the sufficient condition of Lemma 2 is fulfilled in this paper and we proceed considering time-varying channels then.

\section{Online Control Algorithm}

It is worth noticing that problem (15) has long-term timeaverage limitations on power consumption and queuing delay. Using the technique similar to [47], we construct power virtual queue $Y$ and delay virtual queue $Z_{n}$ to track the power consumption and queuing delay respectively. These virtual queues do not exist in practice, and they are just generated by the iterations of (22) and (23):

$$
\begin{aligned}
Y(t+1) & =\left[Y(t)-P_{a v g}\right]^{+}+E(t) \\
Z_{n}(t+1) & =\left[Z_{n}(t)-\rho_{n} \mu_{n}^{o}\right]^{+}+Q_{n}^{o}(t)
\end{aligned}
$$

Similar to actual queues, $Y$ and $Z_{n}$ have initial values of zero. According to Necessary Condition for Rate Stability in [47], if $Y$ is stable, constraint (13) is satisfied. In addition, if $Z_{n}$ is stable, $q_{n}^{o}=\lim _{t \rightarrow \infty} \frac{1}{t} \sum_{\tau=0}^{t-1} \mathbb{E}\left\{Q_{n}^{o}(\tau)\right\} \leq \rho_{n} \nu_{n} \leq \rho_{n} t_{n}^{o}$ holds. According to Little's Theorem, $q_{n}^{o} / t_{n}^{o}=\rho_{n}^{o}$, when $Z_{n}$ is stable, the delay constraint (14) would be achieved. It will be proven that the proposed optimal control algorithm can stabilize these queues in section VI, that is to say the longterm time-average constraints are fulfilled. 
Using virtual queues $X_{n}, Z_{n}$ and $Y$, we decouple problem (15) into two parts: one is flow control algorithm which decides the admission of data, and another is resource allocation algorithm in charge of subcarrier assignment, power allocation and secure transmission control in every slot. All these control actions aim at secondary links and happen in CBS. The whole algorithm is named CBS-side online control algorithm (COCA).

\section{A. Flow control algorithm}

When external data arrives at CBS, CBS will decide whether to admit it according to queue lengthes. Let $V$ be a fixed nonnegative control parameter. Let $q_{\max }^{o} \geq \mu_{\max }$ and $q_{\max }^{p} \geq$ $D_{\max }$ hold. They are actually the deterministic worst case upper bounds of relative queue length to be proven later. The flow control rules of open data and private data are obtained by solving (24) and (25) respectively:

$$
\begin{aligned}
\text { Minimize } & T_{n}^{o}\left[Q_{n}^{o}-q_{\max }^{o}+\mu_{\max }\right] \\
\text { Subject to: } & 0 \leq T_{n}^{o} \leq D_{n}^{o} \\
& \\
\text { Minimize } & T_{n}^{p}\left[Q_{n}^{p}-q_{\max }^{p}+D_{\max }\right] \\
\text { Subject to: } & 0 \leq T_{n}^{p} \leq D_{n}^{p}
\end{aligned}
$$

The corresponding solutions to (24) and (25) are easy to get:

$$
\begin{gathered}
T_{n}^{o}= \begin{cases}0 & \text { if } Q_{n}^{o}-q_{\max }^{o}+\mu_{\max } \geq 0 \\
D_{n}^{o} & \text { otherwise }\end{cases} \\
T_{n}^{p}= \begin{cases}0 & \text { if } Q_{n}^{p}-q_{\max }^{p}+D_{\max } \geq 0 \\
D_{n}^{p} & \text { otherwise }\end{cases}
\end{gathered}
$$

Here we can have an intuitive explanation on flow control rules. They work like valves. When any actual data queue exceeds some threshold, the corresponding valve would turn off and no data would be admitted.

As to virtual variable $\mu_{n}^{o}$ and $\mu_{n}^{p}$, there are also their respective virtual flow control algorithms (28) and (29) so as to update virtual queues $X_{n}^{o}$ and $X_{n}^{p}$ which will play an important role in resource allocation:

$$
\begin{aligned}
\text { Minimize } & \mu_{n}^{o}\left[\frac{q_{\max }^{o}-\mu_{\max }}{q_{\max }^{o}} X_{n}^{o}-\rho_{n} Z_{n}-V \varphi_{n}\right](28) \\
\text { Subject to: } & 0 \leq \mu_{n}^{o} \leq D_{n}^{o} \\
\text { Minimize } & \mu_{n}^{p}\left[\frac{q_{\max }^{p}-D_{\max }}{q_{\max }^{p}} X_{n}^{p}-V \theta_{n}\right] \\
\text { Subject to: } & 0 \leq \mu_{n}^{p} \leq D_{n}^{p}
\end{aligned}
$$

Solutions to (28) and (29) are (30) and (31) respectively:

$$
\mu_{n}^{o}= \begin{cases}0 & \text { if } \quad\left(\frac{q_{\max }^{o}-\mu_{\max }}{q_{\max }^{o}} X_{n}^{o}-\rho_{n} Z_{n}-V \varphi_{n}\right) \geq 0 \\ D_{n}^{o} & \text { otherwise }\end{cases}
$$$$
\mu_{n}^{p}= \begin{cases}0 & \text { if } \quad\left(\frac{q_{\max }^{p}-D_{\max }}{q_{\max }^{p}} X_{n}^{p}-V \theta_{n}\right) \geq 0 \\ D_{n}^{p} & \text { otherwise }\end{cases}
$$

\section{B. Resource allocation algorithm}

The resource allocation policy can be found in solving the following optimization problem.

$$
\begin{aligned}
& \text { Maximize } U\left(\boldsymbol{P}^{S U}, \boldsymbol{\zeta}\right) \\
& \text { Subject to: }(1),(12)
\end{aligned}
$$

where $U\left(\boldsymbol{P}^{S U}, \boldsymbol{\zeta}\right)=\sum_{n=1}^{N}\left(\frac{X_{n}^{o} Q_{n}^{o}}{q_{\max }^{o}} R_{n}^{o}+\frac{X_{n}^{p} Q_{n}^{p}}{q_{\max }^{p}} R_{n}^{p}\right)+$ $Q_{0} R_{0}^{P U}-Y E$.

At the beginning of every slot, all $X_{n}^{o}, X_{n}^{p}, Q_{n}^{o}, Q_{n}^{p}$ and $Y$ can be regarded as constants because they all have been decided in the previous slot. $Q_{0}$ can be estimated by CBS by overhearing PBS feedback. In section VII we propose an imperfect estimation scheme of $Q_{0}$ and compare the performances of perfect and imperfect estimations in simulations. Notice that, the resource allocation is determined at the beginning of every slot and all queues are updated at the end of every slot.

Firstly, we can easily decide the vector $\zeta$ maximizing $U$ by assuming that all elements of $\zeta$ are continuous variables between 0 and 1 and in further discussion, we can get a discrete implementation of $\zeta_{n}$.

We take partial derivative in $U\left(\boldsymbol{P}^{S U}, \boldsymbol{\zeta}\right)$ with respect to $\zeta_{n}$ :

$$
\frac{\partial U\left(\boldsymbol{P}^{S U}, \boldsymbol{\zeta}\right)}{\partial \zeta_{n}}=\left(\frac{X_{n}^{p} Q_{n}^{p}}{q_{\max }^{p}}-\frac{X_{n}^{o} Q_{n}^{o}}{q_{\max }^{o}}\right) \sum_{m=1}^{M} \hat{R}_{n}^{m p}
$$

Observing (33), $\sum_{m=1}^{M} \hat{R}_{n}^{m p}$ is no-negative and $U$ is monotonic in $\zeta_{n}$, and thus the optimality condition of secure transmission control is:

$$
\zeta_{n}^{*}= \begin{cases}1 & \text { if } \quad\left(\frac{X_{n}^{p} Q_{n}^{p}}{q_{\max }^{p}}-\frac{X_{n}^{o} Q_{n}^{o}}{q_{\max }^{o}}\right) \geq 0 \\ 0 & \text { otherwise }\end{cases}
$$

Then we use $\zeta^{*}$ to assign subcarrier and power which is the solution to the following optimization problem PS,

$$
\begin{array}{lll}
\text { PS: } & \text { Maximize } & \widetilde{U}\left(\boldsymbol{P}^{S U}\right) \\
& \text { Subject to: } & (1),(12)
\end{array}
$$

where $\widetilde{U}\left(\boldsymbol{P}^{S U}\right)=U\left(\boldsymbol{P}^{S U}, \boldsymbol{\zeta}^{*}\right)$. PS is a typical Weighted Sum Rate (WSR) maximization problem, and it is difficult to find a global optimum since $\widetilde{U}\left(\boldsymbol{P}^{S U}\right)$ is neither convex nor concave of $\boldsymbol{P}^{S U}$. Obviously, PS has a typical D.C. structure which can be optimally solved by D.C. programming [49]. In [50] there lists a dual decomposition iterative suboptimal algorithm solving this kind of constrained nonconvex problem instead of D.C. programming. In addition, because of the characteristics of OFDMA networks, the duality gap is equal to zero even if PS is nonconvex when the number of subcarriers is close to infinity [51]. So we take a more computationally effective dual method to solve PS and due to space limitation, we give the key steps here only.

We define $R_{n}^{m p}=\zeta_{n}^{*} \hat{R}_{n}^{m p}$ and $R_{n}^{m o}=C_{n}^{m}-R_{n}^{m p}$. Then the Lagrange function of PS is expressed as:

$$
J\left(\delta, \boldsymbol{P}^{S U}\right)=\sum_{m=1}^{M}\left\{\sum_{n=1}^{N}\left[\frac{X_{n}^{o} Q_{n}^{o}}{q_{\max }^{o}} R_{n}^{m o}+\frac{X_{n}^{p} Q_{n}^{p}}{q_{\max }^{p}} R_{n}^{m p}-Y p_{n}^{m}\right]\right.
$$




$$
\left.+Q_{0} R_{0}^{m}\right\}+\delta\left(P_{\max }-E\right)
$$

where $\delta$ is the non-negative Lagrange multiplier for the peak power constraint in problem PS. The dual problem of PS is: $\min _{\delta \geq 0} H(\delta)$, where $H(\delta)=\max _{\boldsymbol{P}^{S U} \geq 0}\left\{J\left(\delta, \boldsymbol{P}^{S U}\right)\right\}$.

When $\delta$ is fixed, we can decide the parameters $\boldsymbol{P}^{S U}$ maximizing the objective of $H(\delta)$. Observing $H(\delta)$, we find that it can be decoupled into $M$ subproblem as:

$$
\begin{aligned}
H(\delta) & =\sum_{m=1}^{M} \max _{\boldsymbol{P}_{S U}^{m}} J_{m}\left(\delta, \boldsymbol{P}_{S U}^{m}\right)+\delta P_{\max } \\
& =\sum_{m=1}^{M} \max _{\boldsymbol{P}_{S U}^{m}} \sum_{n=1}^{N} J_{n}^{m}\left(\delta, p_{n}^{m}\right)+\delta P_{\max }
\end{aligned}
$$

where $\boldsymbol{P}_{S U}^{m}=\left\{p_{n}^{m} \mid 1 \leq n \leq N\right\}, J_{m}\left(\delta, \boldsymbol{P}_{S U}^{m}\right)=$ $\sum_{m=1}^{M} J_{n}^{m}\left(\delta, p_{n}^{m}\right), J_{n}^{m}\left(\delta, p_{n}^{m}\right)=\frac{X_{n}^{o} Q_{n}^{o}}{q_{\text {max }}^{o}} R_{n}^{\text {mo }}+\frac{X_{n}^{p} Q_{n}^{p}}{q_{m a x}^{p}} R_{n}^{m p}-$ $(Y+\delta) p_{n}^{m}+Q_{0} R_{0}^{m}(n)$ and

$$
R_{0}^{m}(n)= \begin{cases}0 & m \in \boldsymbol{\Gamma}_{S U} \\ R_{0}^{m} & m \in \boldsymbol{\Gamma}_{0}^{P U}, \varpi_{n}^{m}=1\end{cases}
$$

For $m \in \boldsymbol{\Gamma}_{S U}$, we can get $p_{n}^{m *}$ by taking partial derivative of $J\left(\delta, \boldsymbol{P}^{S U}\right)$ with respect to $p_{n}^{m}$ and making (38) equal to zero:

$$
\begin{aligned}
& \text { for } m \in \boldsymbol{\Gamma}_{S U}: \\
& \begin{array}{l}
\frac{\partial\left(J\left(\delta, \boldsymbol{P}^{S U}\right)\right)}{\partial p_{n}^{m}}=\frac{X_{n}^{o} Q_{n}^{o}}{q_{\max }^{o}} \frac{1}{\ln 2}\left\{\frac{a_{n}^{m}}{1+p_{n}^{m} a_{n}^{m}}-\zeta_{n}\left[\frac{a_{n}^{m}}{1+p_{n}^{m} a_{n}^{m}}\right.\right. \\
\left.\left.-\frac{b_{n}^{m}}{1+p_{n}^{m} b_{n}^{m}}\right]\right\}+\frac{X_{n}^{p} Q_{n}^{p}}{q_{\max }^{p}} \frac{1}{\ln 2} \zeta_{n}\left[\frac{a_{n}^{m}}{1+p_{n}^{m} a_{n}^{m}}-\frac{b_{n}^{m}}{1+p_{n}^{m} b_{n}^{m}}\right] \\
-(Y+\delta)
\end{array}
\end{aligned}
$$

However, for $m \in \Gamma_{0}^{P U}$, a global optimal solution $p_{n}^{m *}$ maximizing $J_{n}^{m}$ can be got easily by an exhaustive search such as clustering methods or enumerative methods [52] and it is computationally tractable [51], [53].

Substituting (34) and $p_{n}^{m *}$ into $J_{n}^{m}\left(\delta, \boldsymbol{P}^{S U}\right)$, the results are denoted as $J_{n}^{m *}$. For any subcarrier $m$, it will be assigned to the user who has the biggest $J_{n}^{m *}\left(\delta, \boldsymbol{P}^{S U}\right)$. Let $n_{m}^{*}$ be the result of subcarrier $m$ 's assignment which is given by:

$$
n_{m}^{*}=\arg \max _{n} J_{n}^{m}, \forall n \quad \text { and } \quad \varpi_{n}^{m *}= \begin{cases}1 & \text { if } n=n_{m}^{*} \\ 0 & \text { otherwise }\end{cases}
$$

Let $E^{*}=\sum_{n=1}^{N} \sum_{m=1}^{M} p_{n}^{m *} \varpi_{n}^{m *}$. As to the value of $\delta$, we use subgradient method to update it as in (40),

$$
\delta(i+1)=[\delta(i)-\varsigma \triangle \delta(i)]^{+}
$$

where $\triangle \delta(i)=P_{\max }-E^{*}(t, i) . \triangle \delta(i)$ is the subgradient of $H(\delta)$ at $\delta$ and $\varsigma$ is the step size which should be a small positive constant. In addition, index $i$ stands for iteration number. When the subgradient method converges, the resource allocation is completed.

From the above description, we can find some principles of resource allocation.

Remark 1: In (34), both virtual and actual queues of open as well as private data reflect the gap between the corresponding user's demand on data rate and the data rate that the system can provide. Thus, $\frac{X_{n}^{o} Q_{n}^{o}}{q_{m a x}^{o}}$ and $\frac{X_{n}^{p} Q_{n}^{p}}{q_{\text {max }}^{p}}$ can be regarded as the transmission urgency of open data and private data. Only when the transmission urgency of private data exceeds open data, CBS would allocate some resource to transmit private data. Otherwise, CBS would use the user's entire resource to transmit open data due to delay constraint. In PS, it is easy to find that a bigger $Y$ results in less power allocated to every user, which will reduce the system power consumption. Also we let $Q_{0}$ to be the weights of $R_{0}^{P U}$ in PS. It means that if the transmission pressure of PU is high, CBS will allocate less power in subcarrier set $\Gamma_{0}^{P U}$ to avoid causing too much interference on primary link.

Remark 2: In the sub-problem of PS, the transmission power of PBS is assumed to be external variables. Even for the worst case that PBS does not control its transmission power actively, the proposed resource allocation algorithm aims to maximize $Q_{0} R_{0}^{P U}$ in PS by adjusting the interference from the secondary networks to primary networks. Thus, it can be found that the proposed algorithm actually does not affect the energy consumption of primary networks too much.

\section{Control algorithm of Multi-PU case}

Flow control algorithm is the same as (26), (27), (30) and (31).

Resource allocation of multi-PU implementation is the solution to problem MPS:

MPS:

Maximize:

$$
\sum_{n=1}^{N} \frac{X_{n}^{o} Q_{n}^{o}}{q_{\max }^{o}} R_{n}^{o}+\sum_{n=1}^{N} \frac{X_{n}^{p} Q_{n}^{p}}{q_{\max }^{p}} R_{n}^{p}+\sum_{k=1}^{K} Q_{k} R_{k}^{P U}-Y E
$$

Subject to:

(1), (12)

In next section, the algorithm performance with single PU is analysed. It is easy for readers to prove that multi-PU implementation ensures primary data queue stability and furthermore enjoys a similar performance as single PU situation.

\section{Algorithm Performance}

Before the analysis it is necessary to introduce some auxiliary variables. Let $\mathbf{t}^{*}=\left(t_{n}^{p, *}, t_{n}^{o, *}\right)$ be the solution to the following problem:

$$
\begin{array}{rc}
\max _{\mathbf{t}: \mathbf{t} \in \mathbf{\Upsilon}} & \sum_{n=1}^{N} \theta_{n} t_{n}^{p}+\varphi_{n} t_{n}^{o}, \\
\text { Subject to: } & e \leq P_{\text {avg }}
\end{array}
$$

And $\mathbf{t}^{*}(\epsilon)=\left(t_{n}^{p, *}(\epsilon), t_{n}^{o, *}(\epsilon)\right)$ denotes the solution of:

$$
\begin{array}{cc}
\max _{\mathbf{t}: \mathbf{t}+\epsilon \in \Upsilon} & \sum_{n=1}^{N} \theta_{n} t_{n}^{p}+\varphi_{n} t_{n}^{o} \\
\text { Subject to: } & e \leq P_{\text {avg }}
\end{array}
$$

According to [54], it is true that:

$\lim _{\epsilon \rightarrow 0} \sum_{n=1}^{N}\left\{\theta_{n} t_{n}^{p, *}(\epsilon)+\varphi_{n} t_{n}^{o, *}(\epsilon)\right\}=\sum_{n=1}^{N}\left\{\theta_{n} t_{n}^{p, *}+\varphi_{n} t_{n}^{o, *}\right\}$ 
TABLE I

ALGORITHM DESCRIPTIONS

Proposed online control algorithm in timeslot $t$

1) Flow control:

Use (26), (27), (30), (31) to calculate $T_{n}^{o}, T_{n}^{p}, \mu_{n}^{o}$ and $\mu_{n}^{p}$

respectively.

2) Resource allocation:

a) Set the Lagrange multiplier $\delta=\delta_{i n i},\left(\delta_{i n i}\right.$ : An initial value of $\left.\delta\right)$.

b) For each $(n, m)$

i) Use (34) to calculate $\zeta_{n}^{*}$.

ii) Use (38) or exhaustive search to find $p_{n}^{m *}$.

iii) Use (39) to calculate $\varpi_{n}^{m *}$.

c) Use (40) to update $\delta$ and calculate $\triangle \delta(i)$.

d) If $|\triangle \delta(i)|>\triangle \delta_{c}$, goto b), else proceed.

$\left(\triangle \delta_{c}\right.$ : converge condition of $\triangle \delta$ )

3) Update the queues:

Use (6), (7), (8), (9), (22) and (23) to update all queues including $Q_{n}^{o}$,

$Q_{n}^{p}, X_{n}^{o}, X_{n}^{p}, Y, Z_{n}$

The algorithm performance will be listed in Theorem 1 and Theorem 2.

Theorem 1: Employing the proposed algorithm, both actual queues of open data $Q_{n}^{o}(t)$ and private data $Q_{n}^{p}(t)$ in CBS have deterministic worst-case bounds:

$$
Q_{n}^{o}(t) \leq q_{\max }^{o}, Q_{n}^{p}(t) \leq q_{\max }^{p}, \forall t, \forall n
$$

Theorem 2: Given

$$
\begin{aligned}
& q_{\max }^{o}>\mu_{\max }+\frac{C_{\max }^{o}+\mu_{\max }^{2}}{2 \epsilon}, \\
& q_{\max }^{p} \geq D_{\max }+\frac{C_{\max }^{p}{ }^{2}+D_{\max }^{2}}{2 \epsilon}, \\
& \rho_{n}>\frac{q_{\max }^{o}}{\nu_{n}^{o, *}(\epsilon)}, \forall n
\end{aligned}
$$

where $\epsilon$ is positive and can be chosen arbitrarily close to zero. The proposed algorithm performance is bounded by:

$$
\begin{array}{r}
\liminf _{t \rightarrow \infty} \frac{1}{t} \sum_{\tau=0}^{t-1} \sum_{n=1}^{N}\left\{\theta_{n} T_{n}^{p}(\tau)+\varphi_{n} T_{n}^{o}(\tau)\right\} \\
\geq \sum_{n=1}^{N}\left\{\varphi_{n} t_{n}^{o, *}(\epsilon)+\theta_{n} t_{n}^{p, *}(\epsilon)\right\}-\frac{B}{V}
\end{array}
$$

where $B$ is a positive constant independent of $V$ and its expression can be found in appendix B.

In addition, the algorithm also ensures that the long-term time-average sum of PU queue $Q_{0}$ and virtual queues $X_{n}^{o}$, $X_{n}^{p}, Z_{n}, Y$ has an upper bound:

$$
\begin{gathered}
\limsup _{t \rightarrow \infty} \frac{1}{t} \sum_{\tau=0}^{t-1}\left\{\sum_{n=1}^{N}\left(X_{n}^{o}+X_{n}^{p}+Z_{n}\right)+Y+Q_{0}\right\} \\
\leq \frac{B+V \sum_{n=1}^{N}\left\{\left[\theta_{n} t_{n}^{p, *}+\varphi_{n} t_{n}^{o, *}\right]\right\}}{\sigma}
\end{gathered}
$$

where $o \leq \sigma \leq \epsilon$. The proof of Theorem 1 is in appendix A. Theorem 2 and the definition of $\sigma$ can be found in appendix B.
Remark 3 (Network stability): According to the definition of strongly stability as shown in (4), (43) and (48) indicate the stabilities of all queues in the network system. As a result, the network system is stabilized and the long-term timeaverage constraints of delay and power are satisfied. Notice here that $Q_{0}$ 's stability is proved means the PU queue stability constraint is fulfilled. $Q_{0}$ 's stability means that the long-term throughput performance is uninfluenced. In addition, if PU's arrival rates are within the stability region of PU networks, $Q_{0}$ 's stability can be ensured by the proposed scheduling algorithm for any transmission power of PU base station. Therefore, the transmission power of PU network is not affected in this situation. Furthermore, (43) states that all the actual queues of open data and private data have deterministic upper bounds, and this characteristic means that the CBS can accommodate the random arrival packets with finite buffer.

Remark 4 (Optimal throughput performance): (47) states a lower-bound on the weighted throughput that our algorithm can achieve. Since $B$ is a constant independent of $V$, our algorithm would achieve a weighted throughput arbitrarily close to $\sum_{n=1}^{N}\left\{\varphi_{n} t_{n}^{o, *}(\epsilon)+\theta_{n} t_{n}^{p, *}(\epsilon)\right\}$ for some $\epsilon \geq 0$. Furthermore, given any $\epsilon \geq 0$, we can get a better algorithm performance by choosing a larger $V$ without improving the buffer sizes. In addition, as it is shown in (42), when $\epsilon$ tends to zero, our algorithm would achieve a weighted throughput arbitrarily close to $\sum_{n=1}^{N}\left\{\varphi_{n} t_{n}^{o, *}+\theta_{n} t_{n}^{p, *}\right\}$ with a tradeoff in queue length bounds and long-term time-average delay constraints as shown in (44)-(46). Thus we can see that with some certain finite buffer sizes, the proposed algorithm can provide arbitrarily-close-to-optimal performance by choosing $V$, and $V$ 's influence on queue length is shifted from actual queues to virtual queues.

\section{IMPLEMENTATION WITH IMPERFECT ESTIMATION}

CBS needs the information of queue length from primary networks to decide the resource allocation among SUs. [17] considers a situation that queue length information is shared among all the nodes, but in CR environment it is impossible to know the non-cooperative PU's queue information precisely. Compared with getting perfect information about $Q_{k}$, it is more realistic to know the time-average packet arrival rate of PUs. Considering this, in this section, we propose an imperfect estimation of $Q_{k}$ by CBS. And the performance of this estimation will be showed in simulation section. If the PU $k$ is busy, the estimated queue length in CBS is:

$$
\hat{Q}_{k}(t+1)=\left[\hat{Q}_{k}(t)-R_{k}^{P U}(t)\right]^{+}+\left(\lambda_{k}+\iota\right)
$$

where $\iota$ is an over-estimated slack variable to promise primary link stability. CBS can get the precise information when PU is idle by listening to primary link ACK to find that no power is used to transmit PU $k$ 's data packets. In this situation, $\hat{Q}_{k}=$ $Q_{k}=0$ perfectly holds.

As to the control algorithm, we use $\hat{Q}_{k}$ to substitute $Q_{k}$ in resource allocation algorithm. For simplicity, we name this implementation COCA-E (CBS-side online control algorithm with estimated PU queue). 

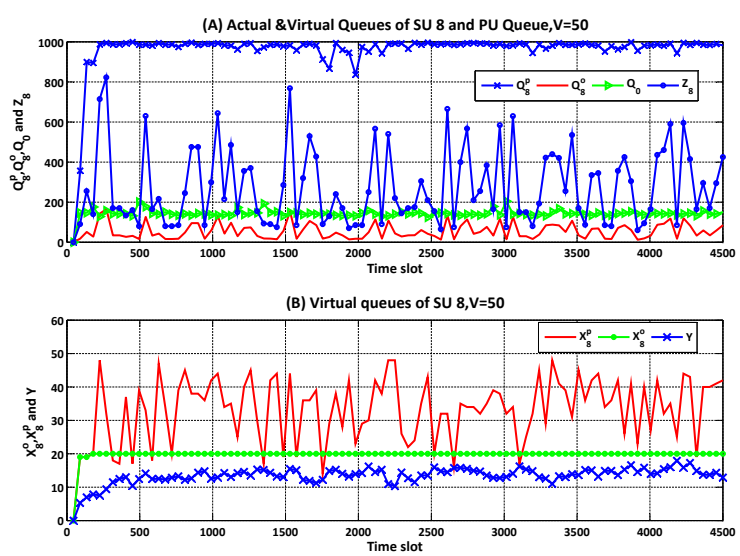

Fig. 3. Queue evolutions over 4500 slots

\section{Simulation}

In this section, we firstly simulate COCA performance in an examplary CR system with a single primary link and secondary network consisting of one CBS, eight SUs and 64 subcarriers. All weights of open data and private data are set to be 0.8 and 1 respectively. The main algorithm parameters of secondary network are set as: $P_{a v g}=0.8 \mathrm{~W}$, $P_{\max }=1 W, \rho_{n}=60, \forall n, q_{\max }^{o}=200, q_{\max }^{p}=1000$, $\mu_{\max }=50, D_{\max }=20$ and $\lambda_{n}^{o}=n * 0.1 * D_{\max }, \lambda_{n}^{p}=$ $n * 0.1 * \mu_{\max }$, for $n \in\{1,2, \cdots, 8\}$. The long-term timeaverage arrival rate of PU $\lambda_{0}$ is set to be 140 and $D_{\max }^{P U}=200$. We simulate the multipath channel of primary and secondary networks as Rayleigh fading channels and the shadowing effect variances are $10 \mathrm{~dB}$. The cross-link channels between PBS to SUs and CBS to PU are simulated as long-scale fading. All parameters in the following parts are set the same as these mentioned here, except for other specification.

In Fig. 3 and Fig. 4, we set average value of $a_{0 S}^{m}, \overline{a_{0 S}}=$ 0.35 , and average value of $a_{n P}^{m}, \overline{a_{n P}}=21, V=50$ and we show both primary and secondary networks' queue evolution over 4500 slots. Because all SUs' data queues $\left(Q_{n}^{o}, Q_{n}^{p}\right)$ and virtual queues $\left(X_{n}^{o}, X_{n}^{p}, Z_{n}\right)$ enjoy similar trends, we take $\mathrm{SU}$ 8 as an example. Fig. 3 (A) shows the dynamics of SU 8's data queues $Q_{8}^{o}, Q_{8}^{p}$, virtual delay queue $Z_{8}$ and PU queue $Q_{0}$. It is observed that both actual data queues are strictly lower than their own deterministic worst case upper bound, which verifies Theorem 1. That $Q_{0}$ is stable in Fig. 3 (A) illustrates that our algorithm can ensure PU queues stability from simulation aspect. Besides, in Fig. 3(B), we can also see that virtual queues $X_{8}^{o}, X_{8}^{p}$ and $Y$ are bounded. So Fig. 3 shows that all queues are bounded, which means that the network system is stabilized and the long-term time-average constraints of delay and power are satisfied.

Fig. 4 directly shows eight SUs' long-term time-average admitted rates and service rates of open data and private data, respectively. Notice that, every user's admitted rate is smaller than service rate and this promises the stabilities of actual data queues.

Fig. 5 shows the relationship between the weighting param-
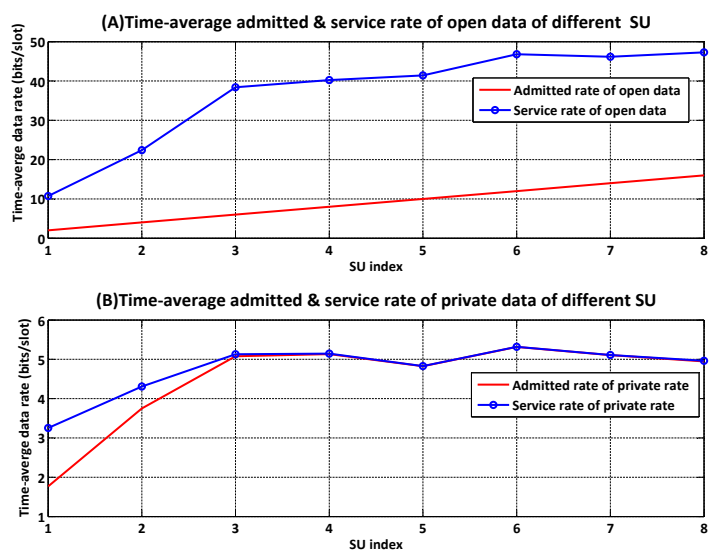

Fig. 4. Long-term time-average admitted and service rate of all SUs

eters and long-term time-average service rates. To show the effects more clearly, we consider the scenario consisting of only one SU and one PU with fixed $\varphi_{1}=450$ and variational $\theta_{1} \in\{0,90.180,270,360,450\}$. The long-term time-average arrival rate of SU is set as: $\lambda_{1}^{p}=8$ and $\lambda_{1}^{o}=260$. The control parameter $V$ is set to be 380 . Each value in Fig. 5 is obtained by averaging the converged results of 5000 times. Fig. 5 shows with the increase of $\theta_{1}$ the long-term time-average service rate of private data increases while the one of open data decreases, which illustrates the effect of throughput weights on long-term time-average service rates.

Fig. 6 demonstrates the relationship between different longterm time-average network performance versus control parameter $V$. In order to compare $\mathrm{PU}$ and SU performance, the similar scenario including one PU and one SU is also considered here. The average data arrival rates of SU are set as: $\lambda_{1}^{o}=250$ and $\lambda_{1}^{p}=10$. In general, the bigger $V$ results in the higher SU open and private transmission rates as Fig. 6 (B) and Fig. 6 (C) respectively show. Fig. 6 (A) demonstrates PU transmission rate decreases as $V$ increases. Notice here, although $r_{0}^{P U}$ decreases, even when $V=380$, $r_{0}^{P U}$ approximates 146 and is greater than $\lambda_{0}=140$, which preserves PU queue stability. Fig. 6 (D) shows the queuing delay performance also improves as $V$ increases.

The implementation of COCA-E with imperfect estimated $Q_{0}$ is simulated. We set the over-estimated slack variable $\iota$ to be 0.01 . We show the differences of the sum service rate of SUs and $R_{0}^{P U}$ between COCA and COCA-E in Fig. 7 (A), Fig. 7 (B) and Fig. 7 (C), respectively. We can see that all the differences are around zero, and SU sum rate is more effected than $R_{0}^{P U}$ by the imperfect estimation of PU queue information. More directly, the influence of $\iota$ on the long-term time-average rate difference between COCA and COCA-E is simulated in Fig. 8, where each record is an averaged result of 1000 converged results. Fig. 8 (C) shows that $r_{0}^{C O C A}-r_{0}^{C O C A-E}$ becomes more negative as $\iota$ increases, which means that the rate decline of PU caused by SU transmissions decreases as $\iota$ increases. More directly, if we want to make sure PU transmission is less influenced, we should choose a larger $\iota$. While a larger $\iota$ inevitably makes 

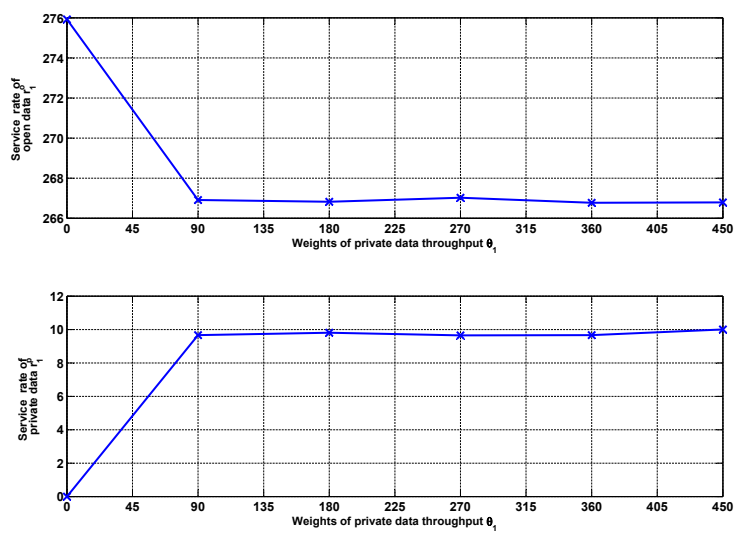

Fig. 5. The time average service rate $\left(r_{1}^{p}\right.$ and $\left.r_{1}^{o}\right)$ versus the weights of private data $\theta_{1}$

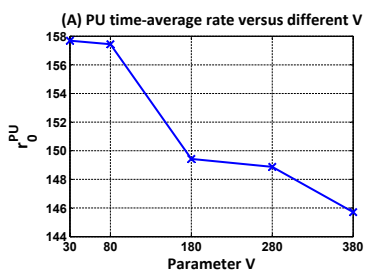
(c) SU time-average private

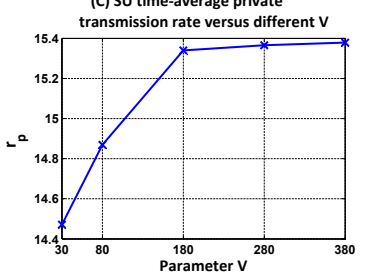

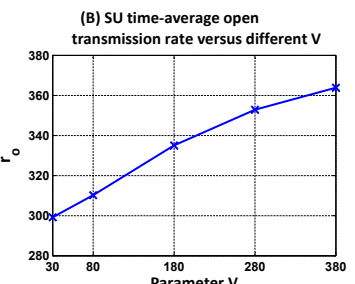

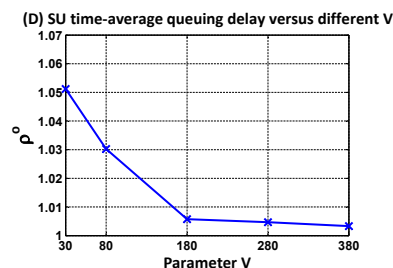

Fig. 6. COCA performances (long-term time-average PU rate, SU rates and SU queuing delay) versus control parameter $V$.

SUs' transmission rates decrease as Fig. 8 (A) and Fig. 8 (B) show.

\section{CONClusions}

In this paper, we propose a cross-layer scheduling and dynamic spectrum access algorithm for maximizing the longterm average throughput of open and private information in an OFDMA-based CR network. We derive the sufficient condition to guarantee that full overlay is optimal in this system. The proposed algorithm can provide a flexible scheduling implementation of open and private information while ensuring the stability of primary networks as well as performance requirements in CR systems with finite buffer size. Furthermore, the proposed algorithm is proved to be close to optimality with current network states in time-varying environments.

\section{APPENDIX A \\ PROOF OF THEOREM 1}

Supposing there exists a slot $t$ satisfying $Q_{n}^{o}(t) \leq q_{\text {max }}^{o}$, it is obviously true for all queues initialized to zero. We prove
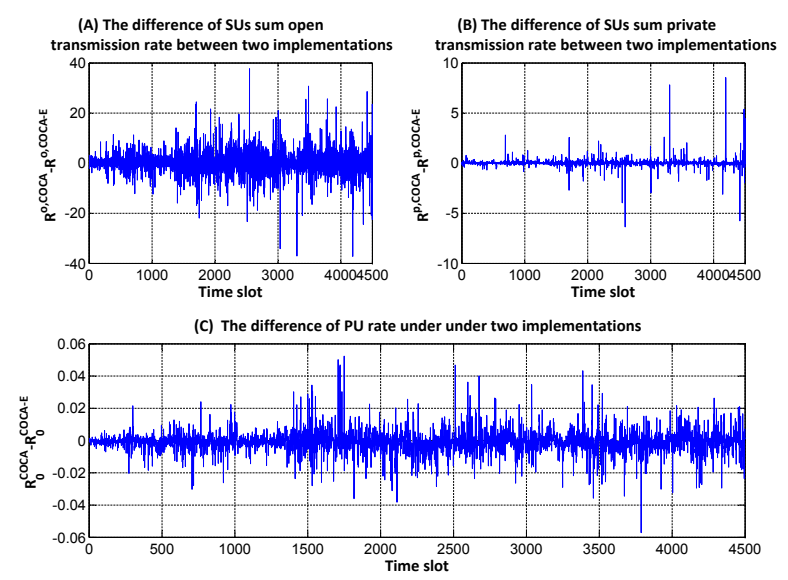

Fig. 7. The rate difference of COCA and COCA-E implementation during 4500 slots.
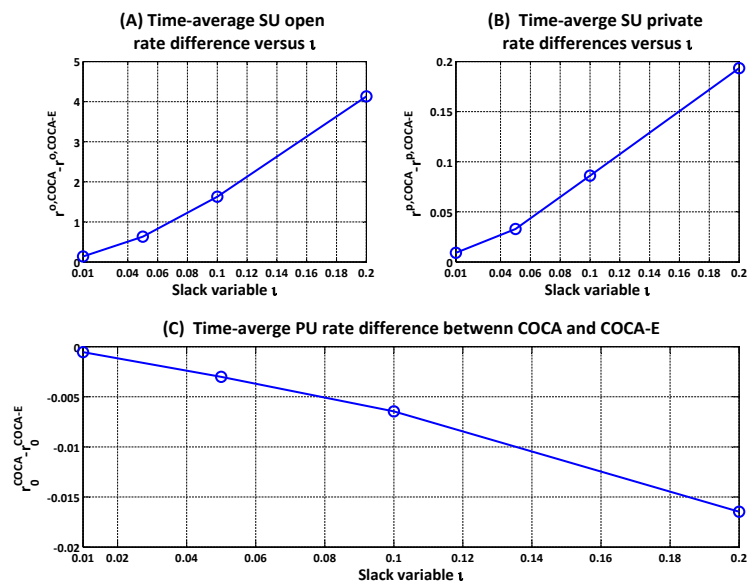

Fig. 8. The long-term time-average rate differences of COCA and COCA-E implementation versus different over-estimated slack variable $\iota$.

that for $t+1$ the same holds. Obviously, there exists two cases. Firstly, we suppose $Q_{n}^{o}(t) \leq q_{\max }^{o}-\mu_{\max }$ and we can easily get $Q_{n}^{o}(t+1) \leq q_{\max }^{o}$. Else, if $Q_{n}^{o}(t)>q_{\max }^{o}-\mu_{\max }$, then according to (26), $T_{n}^{o}(t)=0$. Then

$$
Q_{n}^{o}(t+1) \leq Q_{n}^{o}(t) \leq q_{\max }^{o} .
$$

The proof of $Q_{n}^{p} \leq q_{\max }^{p}$ is similar and omitted here.

\section{APPENDIX B}

\section{PROOF OF THEOREM 2}

Let $\mathbf{Q}=\left\{Q_{0}, Q_{n}^{o}, Q_{n}^{p}, X_{n}^{o}, X_{n}^{p}, Y, Z_{n}\right\}$. We define Lyapunov function $L(\mathbf{Q})$ as:

$$
\begin{aligned}
L(\mathbf{Q})= & \frac{1}{2}\left\{\sum _ { n = 1 } ^ { N } \left[\frac{q_{\max }^{o}-\mu_{\max }}{q_{\max }^{o}} X_{n}^{o 2}+Z_{n}^{2}+\frac{1}{q_{\max }^{o}} Q_{n}^{o 2}(t) X_{n}^{o}+\right.\right. \\
& \left.\left.\frac{q_{\max }^{p}-D_{\max }}{q_{\max }^{p}} X_{n}^{p 2}+\frac{1}{q_{\max }^{p}} Q_{n}^{p 2} X_{n}^{p}\right]+Y^{2}+Q_{0}^{2}\right\}
\end{aligned}
$$

According to [47], $\triangle L(\mathbf{Q})$ is defined as the conditional Lyapunov drift for slot $t$ :

$$
\triangle L(\mathbf{Q}) \triangleq \mathbb{E}\{L(\mathbf{Q}(t+1))-L(\mathbf{Q}(t)) \mid \mathbf{Q}(t)\}
$$


According to $(|x-y|+z)^{2} \leq x^{2}+y^{2}+z^{2}-2 x(y-z)$, we can get the results below:

$$
\begin{aligned}
& \frac{q_{\max }^{o}-\mu_{\max }}{q_{\max }^{o}}\left[X_{n}^{o 2}(t+1)-X_{n}^{o 2}(t)\right] \leq \\
& \quad \frac{q_{\max }^{o}-\mu_{\max }}{q_{\max }^{o}}\left\{2 \mu_{\max }^{2}-2 X_{n}^{o}(t)\left[T_{n}^{o}(t)-\mu_{n}^{o}(t)\right]\right\} \\
& \frac{\left[Q_{n}^{o^{2}}(t+1) X_{n}^{o}(t+1)-Q_{n}^{o^{2}}(t) X_{n}^{o}(t)\right]}{q_{\max }^{o}} \leq q_{\max }^{o} \mu_{\max }+ \\
& \frac{\left(\mu_{\max }^{2}+C_{\max }^{o^{2}}\right)-2 Q_{n}^{o}(t)\left[R_{n}^{o}(t)-T_{n}^{o}(t)\right]}{q_{\max }^{o}} X_{n}^{o}(t)
\end{aligned}
$$

The queues of private data have similar inequalities above. Furthermore, we can derive that:

$$
\begin{aligned}
& \triangle L(\mathbf{Q})-V \sum_{n=1}^{N} \mathbb{E}\left\{\theta_{n} \mu_{n}^{p}+\varphi_{n} \mu_{n}^{o} \mid \mathbf{Q}\right\} \leq B-Q_{0} \mathbb{E}\left\{R_{0}^{P U}-\right. \\
& \left.D_{0}^{P U} \mid \mathbf{Q}\right\}-Y \mathbb{E}\left\{P_{\text {avg }}-E \mid \mathbf{Q}\right\}+\sum_{n=1}^{N}\left\{\frac{X_{n}^{o}\left(C_{\max }^{o^{2}}+\mu_{\max }^{2}\right)}{2 q_{\max }^{o}}\right. \\
& +\frac{X_{n}^{p}\left(C_{\max }^{p^{2}}+D_{\max }^{2}\right)}{2 q_{\max }^{p}}-\frac{X_{n}^{p} Q_{n}^{p}}{q_{\max }^{p}} \mathbb{E}\left\{R_{n}^{p}-T_{n}^{p} \mid \mathbf{Q}\right\}- \\
& \frac{X_{n}^{o} Q_{n}^{o}}{q_{\max }^{o}} \mathbb{E}\left\{R_{n}^{o}-T_{n}^{o} \mid \mathbf{Q}\right\}-\left(1-\frac{D_{\max }}{q_{\max }^{p}}\right) X_{n}^{p} \mathbb{E}\left\{T_{n}^{p}-\mu_{n}^{p} \mid \mathbf{Q}\right\}- \\
& \left(1-\frac{\mu_{\max }}{q_{\max }^{o}}\right) X_{n}^{o} \mathbb{E}\left\{T_{n}^{o}-\mu_{n}^{o} \mid \mathbf{Q}\right\}-Z_{n} \mathbb{E}\left\{\rho_{n} \mu_{n}^{o}-Q_{n}^{o} \mid \mathbf{Q}\right\} \\
& \left.-V \mathbb{E}\left\{\theta_{n} \mu_{n}^{p}+\varphi_{n} \mu_{n}^{o} \mid \mathbf{Q}\right\}\right\}
\end{aligned}
$$

where $B=\frac{1}{2}\left(D_{\max }^{P U^{2}}+R_{0 \max }^{2}+P_{\max }^{2}+P_{a v g}^{2}\right)+$ $N\left[\frac{1}{2} q_{\max }^{o} \mu_{\max }+\left(1-\frac{\mu_{\max }}{q_{\max }^{o}}\right) \mu_{\max }^{2}+\left(1-\frac{D_{\max }}{q_{\max }^{p}}\right) D_{\max }^{2}+\right.$ $\left.\frac{1}{2} q_{\max }^{p} D_{\max }\right]+\frac{1}{2} \sum_{n=1}^{N}\left(\rho_{n}^{2} \mu_{\max }^{2}+q_{\max }^{o^{2}}\right)$ and $C_{\max }^{p}=$ $\max _{n}\left\{R_{n}^{p}\right\}, C_{\max }^{o}=\max _{n}\left\{R_{n}^{o}\right\}, R_{0 \max }=\max \left\{R_{0}^{P U}\right\}$. Here we can find that our algorithm minimizes the right hand side (RHS) of (54).

In order to prove Theorem 2, we introduce Lemma 3.

Lemma 3: For any feasible rate vector $\mathbf{t} \in \boldsymbol{\Upsilon}$, there exists a a-only policy $S R$ which stabilizes the network with the data admitted rate vector, $\left(\mu_{n, S R}^{p}(t), t_{n, S R}^{p}(t), \mu_{n, S R}^{o}(t), t_{n, S R}^{o}(t)\right)$, and the service vector, $\left(R_{n, S R}^{p}(t), R_{n, S R}^{o}(t)\right)$, independent of data queues. For all $t$ and all $n \in\{1,2, \ldots, N\}$, the flow constraints are satisfied:

$$
\begin{aligned}
& \mathbb{E}\left\{\mu_{n, S R}^{o}(t)\right\}=\mathbb{E}\left\{T_{n, S R}^{o}(t)\right\}=\mathbb{E}\left\{R_{n, S R}^{o}\right\} \\
& \mathbb{E}\left\{\mu_{n, S R}^{p}(t)\right\}=\mathbb{E}\left\{T_{n, S R}^{p}(t)\right\}=\mathbb{E}\left\{R_{n, S R}^{p}\right\}
\end{aligned}
$$

Notice that, the stationary randomized policy $S R$ makes decisions only depending on channel condition and independent of queue backlogs. Furthermore it may not fulfill the delay constraints. Similar proof of a-only policy is given in [17] and the proof of Lemma 3 is omitted here.

We can control the admitted rate of $\mathbf{t}$ ranging from $\mathbf{t}^{*}(\epsilon)$ to $\mathbf{t}^{*}(\epsilon)+\epsilon$ arbitrarily and resulting in that both $\mathbf{t}^{*}(\epsilon)$ and $\mathbf{t}^{*}(\epsilon)+$ $\epsilon$ are within $\Upsilon$. It is assumed that the sufficient condition of full overlay optimum (21) is satisfied in our system, so according to Lemma 2, full overlay can achieve the optimal result. Besides, according to Lemma 3, it is true that there exist two different a-only policies $S R_{1}$ and $S R_{2}$ which satisfy:

$$
\begin{aligned}
& \mathbb{E}\left\{T_{n, S R_{1}}^{o}\right\}=\mathbb{E}\left\{R_{n, S R_{1}}^{o}\right\}=\mathbb{E}\left\{\mu_{n, S R_{1}}^{o}\right\}=t_{n}^{o, *}(\epsilon) \\
& \mathbb{E}\left\{T_{n, S R_{1}}^{p}\right\}=\mathbb{E}\left\{R_{n, S R_{1}}^{p}\right\}=\mathbb{E}\left\{\mu_{n, S R_{1}}^{p}\right\}=t_{n}^{p, *}(\epsilon) \\
& \mathbb{E}\left\{T_{n, S R_{2}}^{o}\right\}=\mathbb{E}\left\{R_{n, S R_{2}}^{o}\right\}=\mathbb{E}\left\{\mu_{n, S R_{2}}^{o}\right\}=t_{n}^{o, *}(\epsilon)+\epsilon \\
& \mathbb{E}\left\{T_{n, S R_{2}}^{p}\right\}=\mathbb{E}\left\{R_{n, S R_{2}}^{p}\right\}=\mathbb{E}\left\{\mu_{n, S R_{2}}^{p}\right\}=t_{n}^{p, *}(\epsilon)+\epsilon
\end{aligned}
$$

In addition, for policy $S R_{1}$ and $S R_{2}$, it is easy to prove that:

$$
\begin{aligned}
& \mathbb{E}\left\{R_{0, S R_{1}}^{P U}\right\} \geq \lambda_{0}+\epsilon \\
& \mathbb{E}\left\{E_{S R_{2}}\right\} \leq P_{\text {avg }}-\epsilon
\end{aligned}
$$

Our algorithm minimizes RHS of (54) among all possible policies including $S R$ policy, thus we can get :

$$
\begin{aligned}
& \triangle L(\mathbf{Q})-V \sum_{n=1}^{N} \mathbb{E}\left\{\theta_{n} \mu_{n}^{p}+\varphi_{n} \mu_{n}^{o}\right\} \leq B+ \\
& Y\left\{\mathbb{E}\left\{E_{S R_{2}}\right\}-P_{\text {avg }}\right\}-Q_{0}\left\{\mathbb{E}\left\{R_{0, S R_{1}}^{P U}-\lambda_{0}\right\}+\right. \\
& \sum_{n=1}^{N}\left\{Z_{n} Q_{n}^{o}+\frac{C_{\text {max }}^{o^{2}}+\mu_{\max }^{2}}{2 q_{\max }^{o}} X_{n}^{o}+\frac{C_{\max }^{p^{2}}+D_{\max }^{2}}{2 q_{\max }^{p}} X_{n}^{p}+\right. \\
& {\left[\frac{q_{\max }^{o}-\mu_{\max }}{q_{\max }^{o}} X_{n}^{o}-Z_{n} \rho_{n}-V \varphi_{n}\right] X_{n}^{o} \mathbb{E}\left\{\mu_{n, S R_{1}}^{o}\right\}+} \\
& \mathbb{E}\left\{T_{n, S R_{2}}^{o}\right\} \frac{X_{n}^{o}}{q_{\max }^{o}}\left[Q_{n}^{o}+\mu_{\max }-q_{\max }^{o}\right]-\frac{X_{n}^{o} Q_{n}^{o}}{q_{\max }^{o}} \mathbb{E}\left\{R_{n, S R_{2}}^{o}\right\}+ \\
& \mathbb{E}\left\{T_{n, S R_{2}}^{p}\right\} \frac{X_{n}^{p}}{q_{\max }^{p}}\left[Q_{n}^{p}-q_{\max }^{p}+D_{\max }\right]-\frac{X_{n}^{p} Q_{n}^{p}}{q_{\max }^{p}} \mathbb{E}\left\{R_{n, S R_{2}}^{p}\right\}+ \\
& \left.\mathbb{E}\left\{\mu_{n, S R_{1}}^{p}\right\}\left[\frac{q_{\max }^{p}-D_{\max }}{q_{\max }^{p}} X_{n}^{p}-V \theta_{n}\right]\right\}
\end{aligned}
$$

After substituting (55)-(58), (59) and (60) into the RHS of (61) and transforming it, we can derive that:

$$
\begin{aligned}
& \triangle L(Q)-V \sum_{n=1}^{N} \mathbb{E}\left\{\theta_{n} \mu_{n}^{p}+\varphi_{n} \mu_{n}^{o}\right\} \leq B-\epsilon\left(Y+Q_{0}\right)- \\
& \sum\left\{t_{n}^{o, *}(\epsilon) \rho-q_{\max }\right\} Z-V \sum_{n=1}^{N}\left\{\varphi_{n} t_{n}^{o, *}(\epsilon)+\theta_{n} t_{n}^{p, *}(\epsilon)\right\}- \\
& \sum_{n=1}^{N} \frac{X_{n}^{o}}{q_{\text {max }}^{o}}\left\{\epsilon\left(q_{\text {max }}^{o}-\mu_{\max }\right)-\frac{C_{\max }^{o^{2}}+\mu_{\max }^{2}}{2}\right\}- \\
& \sum_{n=1}^{N} \frac{X_{n}^{p}}{q_{\max }^{p}}\left\{\epsilon\left(q_{\max }^{p}-D_{\max }\right)-\frac{C_{\max }^{p^{2}}+D_{\max }^{2}}{2}\right\}
\end{aligned}
$$

So when (44)-(46) hold, we can find $\epsilon_{1}>0$ that $\epsilon_{1} \leq$ $\frac{\epsilon\left(q_{\max }^{o}-\mu_{\max }\right)-\frac{C_{\max }^{o^{2}}+\mu_{\max }^{2}}{2}}{q_{\max }^{o}}, \epsilon_{1} \leq t_{n}^{o, *}(\epsilon) \rho_{n}-q_{\max }$ and $\epsilon_{1} \leq$ $\frac{\epsilon\left(q_{\max }^{p}-D_{\max }\right)-\frac{C_{\max }^{p^{2}}+D_{\max }^{2}}{2}}{q_{\max }^{p}}$. Thus:

$$
\begin{aligned}
& \triangle L(Q)-V \sum_{n=1}^{N} \mathbb{E}\left\{\theta_{n} \mu_{n}^{p}+\varphi_{n} \mu_{n}^{o}\right\} \leq B-V \sum_{n=1}^{N}\left\{\varphi t_{n}^{o, *}(\epsilon)+\right. \\
& \left.\theta_{n} t_{n}^{p, *}(\epsilon)\right\}-\sigma\left(\sum_{n=1}^{N}\left\{X_{n}^{o}+X_{n}^{p}+Z_{n}\right\}+Y+Q_{0}\right)
\end{aligned}
$$


where $\sigma=\min \left\{\epsilon, \epsilon_{1}\right\}$.

It can be got that when (44), (45) and (46) hold, (48) and

$$
\begin{gathered}
\liminf _{t \rightarrow \infty} \frac{1}{t} \sum_{\tau=0}^{t-1} \sum_{n=1}^{N}\left\{\theta_{n} \mu_{n}^{p}(\tau)+\varphi_{n} \mu_{n}^{o}(\tau)\right\} \geq \\
\sum_{n=1}^{N}\left\{\varphi_{n} t_{n}^{o, *}(\epsilon)+\theta_{n} t_{n}^{p, *}(\epsilon)\right\}-\frac{B}{V}
\end{gathered}
$$

are satisfied by applying the theorem of Lyapunov Optimization, Theorem 4.2 in [47], on (63) directly. Furthermore, (48) implies that (10) and (11) hold since $X_{n}^{o}$ and $X_{n}^{p}$ are kept stable. So after substituting (10) and (11) into (64), (47) holds. Hence the proof of Theorem 2 is completed.

\section{REFERENCES}

[1] T. A. Weiss and F. K. Jondral, "Spectrum pooling: an innovative strategy for the enhancement of spectrum efficiency," Communications Magazine, IEEE, vol. 42, no. 3, pp. S8-14, 2004.

[2] X. Huang, D. Lu, P. Li, and Y. Fang, "Coolest path: spectrum mobility aware routing metrics in cognitive ad hoc networks," in Distributed Computing Systems (ICDCS), 2011 31st International Conference on. IEEE, 2011, pp. 182-191.

[3] R. Deng, J. Chen, X. Cao, Y. Zhang, S. Maharjan, and S. Gjessing, "Sensing-performance tradeoff in cognitive radio enabled smart grid," Smart Grid, IEEE Transactions on, vol. 4, no. 1, pp. 302-310, March 2013.

[4] R. Deng, J. Chen, C. Yuen, P. Cheng, and Y. Sun, "Energy-efficient cooperative spectrum sensing by optimal scheduling in sensor-aided cognitive radio networks," Vehicular Technology, IEEE Transactions on, vol. 61, no. 2, pp. 716-725, Feb 2012.

[5] E. Lawrey, "Multiuser ofdm," in Signal Processing and Its Applications, 1999. ISSPA'99. Proceedings of the Fifth International Symposium on, vol. 2. IEEE, 1999, pp. 761-764.

[6] X. Zhou, G. Y. Li, and G. Sun, "Multiuser spectral precoding for ofdm-based cognitive radios," in Global Telecommunications Conference (GLOBECOM 2011), 2011 IEEE. IEEE, 2011, pp. 1-5.

[7] - "Low-complexity spectrum shaping for ofdm-based cognitive radios," in Wireless Communications and Networking Conference (WCNC), 2011 IEEE. IEEE, 2011, pp. 1471-1475.

[8] S. M. Almalfouh and G. L. Stuber, "Interference-aware radio resource allocation in ofdma-based cognitive radio networks," Vehicular Technology, IEEE Transactions on, vol. 60, no. 4, pp. 1699-1713, 2011.

[9] Y. Zhang and C. Leung, "Resource allocation for non-real-time services in ofdm-based cognitive radio systems," Communications Letters, IEEE, vol. 13, no. 1, pp. 16-18, 2009.

[10] _ - "Cross-layer resource allocation for mixed services in multiuser ofdm-based cognitive radio systems," Vehicular Technology, IEEE Transactions on, vol. 58, no. 8, pp. 4605-4619, 2009.

[11] B. Wang and K. Liu, "Advances in cognitive radio networks: A survey," Selected Topics in Signal Processing, IEEE Journal of, vol. 5, no. 1, pp. 5-23, 2011.

[12] Q. Zhao and B. M. Sadler, "A survey of dynamic spectrum access," Signal Processing Magazine, IEEE, vol. 24, no. 3, pp. 79-89, 2007.

[13] J. Huang, R. A. Berry, and M. L. Honig, "Spectrum sharing with distributed interference compensation," in New Frontiers in Dynamic Spectrum Access Networks, 2005. DySPAN 2005. 2005 First IEEE International Symposium on. IEEE, 2005, pp. 88-93.

[14] L. Le and E. Hossain, "Qos-aware spectrum sharing in cognitive wireless networks," in Global Telecommunications Conference, 2007. GLOBECOM'07. IEEE. IEEE, 2007, pp. 3563-3567.

[15] M. Levorato, U. Mitra, and M. Zorzi, "Cognitive interference management in retransmission-based wireless networks," Information Theory, IEEE Transactions on, vol. 58, no. 5, pp. 3023-3046, 2012.

[16] S. Huang, X. Liu, and Z. Ding, "Distributed power control for cognitive user access based on primary link control feedback," in INFOCOM, 2010 Proceedings IEEE. IEEE, 2010, pp. 1-9.

[17] L. Georgiadis, M. J. Neely, and L. Tassiulas, "Resource allocation and

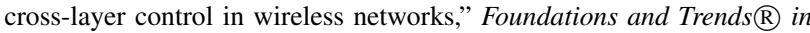
Networking, vol. 1, no. 1, pp. 1-144, 2006.

[18] F. E. Lapiccirella, X. Liu, and Z. Ding, "Distributed control of multiple cognitive radio overlay for primary queue stability," IEEE transactions on wireless communications, vol. 12, no. 1, pp. 112-122, 2013.
[19] J. Jang and K. Lee, "Transmit power adaptation for multiuser ofdm systems," Selected Areas in Communications, IEEE Journal on, vol. 21 no. 2, pp. 171-178, 2003.

[20] S. W. Kim, B.-S. Kim, and Y. Fang, "Downlink and uplink resource allocation in ieee 802.11 wireless lans," Vehicular Technology, IEEE Transactions on, vol. 54, no. 1, pp. 320-327, 2005.

[21] K. Seong, M. Mohseni, and J. Cioffi, "Optimal resource allocation for ofdma downlink systems," in Information Theory, 2006 IEEE International Symposium on. IEEE, 2006, pp. 1394-1398.

[22] Y. Zou, T. Chen, and S. Li, "Network-based predictive control of multirate systems," IET control theory \& applications, vol. 4, no. 7, pp. 1145-1156, 2010.

[23] X. Zhu, J. Yue, B. Yang, and X. Guan, "Flow rate control and resource allocation policy with security requirements in ofdma networks," in Intelligent Control and Automation (WCICA), 2012 10th World Congress on. IEEE, 2012, pp. 1020-1025.

[24] Z. Shen, J. Andrews, and B. Evans, "Adaptive resource allocation in multiuser ofdm systems with proportional rate constraints," Wireless Communications, IEEE Transactions on, vol. 4, no. 6, pp. 2726-2737, 2005.

[25] G. Li and H. Liu, "Dynamic resource allocation with finite buffer constraint in broadband ofdma networks," in Wireless Communications and Networking, 2003. WCNC 2003. 2003 IEEE, vol. 2. IEEE, 2003, pp. 1037-1042.

[26] X. Huang and Y. Fang, "Multiconstrained qos multipath routing in wireless sensor networks," Wireless Networks, vol. 14, no. 4, pp. 465478, 2008.

[27] Y. Cui, V. Lau, R. Wang, H. Huang, and S. Zhang, "A survey on delay-aware resource control for wireless systemsłlarge deviation theory, stochastic lyapunov drift, and distributed stochastic learning," Information Theory, IEEE Transactions on, vol. 58, no. 3, pp. 1677-1701, 2012.

[28] Z. Yuanyuan, L. Shaoyuan, and N. Yugang, "Networked predictive control of constrained linear systems with stability guarantee," in Control Conference (CCC), 2010 29th Chinese, July 2010, pp. 4355-4360.

[29] D. Xue and E. Ekici, "Delay-guaranteed cross-layer scheduling in multihop wireless networks," arXiv preprint arXiv:1009.4954, 2010.

[30] R. Urgaonkar and M. Neely, "Delay-limited cooperative communication with reliability constraints in wireless networks," in INFOCOM 2009, IEEE. IEEE, 2009, pp. 2561-2565.

[31] C. Shannon, "Communication theory of secrecy systems," Bell system technical journal, vol. 28, no. 4, pp. 656-715, 1949.

[32] L. Ozarow and A. Wyner, "Wire-tap channel ii," in Advances in Cryptology. Springer, 1985, pp. 33-50.

[33] D. W. K. Ng, E. S. Lo, and R. Schober, "Energy-efficient resource allocation for secure ofdma systems," Vehicular Technology, IEEE Transactions on, vol. 61, no. 6, pp. 2572-2585, 2012.

[34] X. Wang, M. Tao, J. Mo, and Y. Xu, "Power and subcarrier allocation for physical-layer security in ofdma-based broadband wireless networks," Information Forensics and Security, IEEE Transactions on, vol. 6, no. 3, pp. 693-702, 2011.

[35] Y. Pei, Y.-C. Liang, L. Zhang, K. C. Teh, and K. H. Li, "Secure communication over miso cognitive radio channels," Wireless Communications, IEEE Transactions on, vol. 9, no. 4, pp. 1494-1502, 2010.

[36] T. Kwon, V. W. Wong, and R. Schober, "Secure miso cognitive radio system with perfect and imperfect csi," in Global Communications Conference (GLOBECOM), 2012 IEEE. IEEE, 2012, pp. 1236-1241.

[37] Y. Liang, A. Somekh-Baruch, H. V. Poor, S. Shamai, and S. Verdú, "Capacity of cognitive interference channels with and without secrecy," Information Theory, IEEE Transactions on, vol. 55, no. 2, pp. 604-619, 2009.

[38] E. Ekrem and S. Ulukus, "Capacity region of gaussian mimo broadcast channels with common and confidential messages," Information Theory, IEEE Transactions on, vol. 58, no. 9, pp. 5669-5680, 2012.

[39] X. Zhu, B. Yang, and X. Guan, "Cross-layer scheduling with secrecy demands in delay-aware ofdma network." in Wireless Communications and Networking Conference (WCNC), 2013 IEEE. IEEE, 2013, pp. 1339-1344.

[40] D. Tse and P. Viswanath, Fundamentals of wireless communication. Cambridge university press, 2005.

[41] M. Wallace, J. R. Walton, and A. Jalali, "Method and apparatus for measuring reporting channel state information in a high efficiency, high performance communications system," Oct. 29 2002, uS Patent $6,473,467$.

[42] H. A. Suraweera, P. J. Smith, and M. Shafi, "Capacity limits and performance analysis of cognitive radio with imperfect channel knowledge," Vehicular Technology, IEEE Transactions on, vol. 59, no. 4, pp. 1811$1822,2010$. 
[43] S. McLaughlin, W. Harrison, J. McConnell, and C. Argon, "Applications for physical-layer security," Jun. 19 2014, uS Patent App. 13/962,777. [Online]. Available: https://www.google.com/patents/US20140171856

[44] S. W. McLaughlin, D. Klinc, B.-J. Kwak, and D. S. Kwon, "Secure communication using error correction codes," Jul. 9 2013, uS Patent $8,484,545$.

[45] C. Argon, "Pre-processor for physical layer security."

[46] C. Koksal, O. Ercetin, and Y. Sarikaya, "Control of wireless networks with secrecy," in Signals, Systems and Computers (ASILOMAR), 2010 Conference Record of the Forty Fourth Asilomar Conference on. IEEE, 2010, pp. 47-51.

[47] M. Neely, "Stochastic network optimization with application to communication and queueing systems," Synthesis Lectures on Communication Networks, vol. 3, no. 1, pp. 1-211, 2010.

[48] X. Zhu, B. Yang, C. Chen, L. Xue, X. Guan, and F. Wu, "Crosslayer scheduling for ofdma-based cognitive radio systems with delay and security constraints," arXiv:1412.8375, 2014.

[49] Y. Xu, T. Le-Ngoc, and S. Panigrahi, "Global concave minimization for optimal spectrum balancing in multi-user dsl networks," Signal Processing, IEEE Transactions on, vol. 56, no. 7, pp. 2875-2885, 2008

[50] L. Venturino, N. Prasad, and X. Wang, "Coordinated scheduling and power allocation in downlink multicell ofdma networks," Vehicular Technology, IEEE Transactions on, vol. 58, no. 6, pp. 2835-2848, 2009.

[51] W. Yu and R. Lui, "Dual methods for nonconvex spectrum optimization of multicarrier systems," Communications, IEEE Transactions on, vol. 54, no. 7, pp. 1310-1322, 2006.

[52] R. Horst and H. Tuy, Global optimization: Deterministic approaches. Springer, 1996.

[53] R. Cendrillon, W. Yu, M. Moonen, J. Verlinden, and T. Bostoen, "Optimal multiuser spectrum balancing for digital subscriber lines," Communications, IEEE Transactions on, vol. 54, no. 5, pp. 922-933, 2006.

[54] A. Stolyar, "Maximizing queueing network utility subject to stability: Greedy primal-dual algorithm," Queueing Systems, vol. 50, no. 4, pp 401-457.

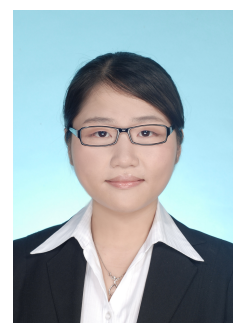

Xingzheng Zhu received the B.S. degree in $\mathrm{Au}-$ tomation from Harbin Institute of Technology, Harbin, China, in 2011, the M.S. degree in Control Science and Control Engineering from Shanghai Jiao Tong University, Shanghai, China, in 2014. She is currently pursuing a Ph.D. degree in the Department of Electrical and Electronic Engineering, The University of Hong Kong. Her research interests include wireless communication networks and smart grids.

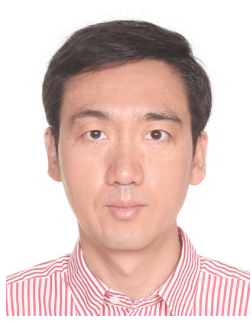

Bo Yang (M'09) obtained his PhD in Electrical Engineering from the City University of Hong Kong in 2009. Prior to joining Shanghai Jiao Tong University in 2010, he was a postdoctoral researcher at the Royal Institute of Technology (KTH), Sweden, from 2009 to 2010 and a visiting scholar at the Polytechnic Institute of New York University in 2007. $\mathrm{He}$ is now an associate professor in Shanghai Jiao Tong University. He is also a cyber scholar with the Cyber Joint Innovation Center founded by Zhejiang University, Tsinghua University, and Shanghai Jiao

Tong University.

His current research interest includes game theoretical analysis and nonlinear optimization of communication networks and smart grid. He is on the editorial board of Digital Signal Processing-Elsevier and in the TPC of several international conferences. Dr. Yang has been the principle/co-investigator in several research projects funded by NSF of China, Swedish Governmental Agency for Innovation Systems, and the US air force. He is a Member of IEEE and ACM.

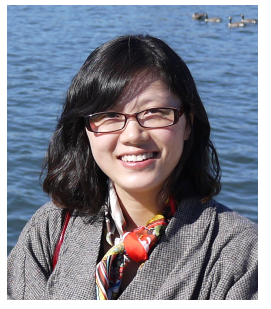

Cailian Chen (S'03-M'06) received the B.Eng. and M.Eng. degrees in Automatic Control from Yanshan University, China in 2000 and 2002, respectively, and the Ph.D. degree in Control and Systems from City University of Hong Kong, Hong Kong SAR in 2006. She joined Department of Automation, Shanghai Jiao Tong University in 2008 as an Associate Professor. She is now a Full Professor.

Dr. Chen's research interests include Distributed Estimation and Control of Network Systems, Wireless Sensor and Actuator Network, Multi-agent Systems and Intelligent Control Systems. She has authored and/or coauthored 2 research monographs and over 80 referred international journal and conference papers. She is the inventor of 20 patents. Dr. Chen received the "IEEE Transactions on Fuzzy Systems Outstanding Paper Award" in 2008. She was one of the First Prize Winners of Natural Science Award from The Ministry of Education of China in 2007. She was honored as "New Century Excellent Talents in University of China" and "Shanghai Rising Star" in 2013, "Shanghai Pujiang Scholar", "Chenguang Scholar" and "SMC Outstanding Young Staff of Shanghai Jiao Tong University" in 2009.

She is a Member of IEEE. She serves as an Associate Editor of Peertopeer Networking and Applications (Springer), ISRN Sensor Networks, and Scientific World Journal (Computer Science), and TPC member of several flagship conferences including IEEE Globecom, IEEE ICC and IEEE WCCI.

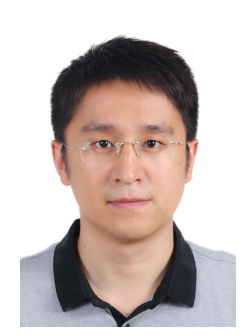

Liang Xue received the B.S., M.S., and Ph.D. degrees in control theory and engineering from Yanshan University, Qinhuangdao, China, in 2006, 2009, and 2012, respectively. He is currently an associate professor with the School of Information and Electrical Engineering and the chair of the Department of Internet of Things, Hebei University of Engineering, Handan, China. He is also the Outstanding Young Scholar of Hebei Education Department and Hebei new century " 333 talent project " third level suitable person. He is now in charge of several research projects including the National Natural Science Foundation of China, the Scientific Research Plan of Hebei Education Department, etc. His research interests include the clustering design, hierarchical topology control, and data routing in wireless sensor networks and wireless cognitive radio networks.

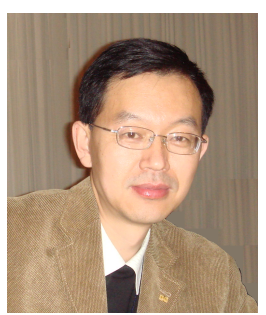

Xinping Guan (M'02-SM'04) received the Ph.D. degree in control and systems from Harbin Institute of Technology, Harbin, China in 1999. In 2007, he joined the Department of Automation, Shanghai Jiao Tong University, Shanghai, China, where he is currently a Distinguished University Professor, the Executive Deputy Dean of University Office of Research Management, and the Director of the Key Laboratory of Systems Control and Information Processing, Ministry of Education of China. Before that, he was the Professor and Dean of Electrical Engineering, Yanshan University, China in 1998-2008.

Dr. Guan's current research interests include cyber-physical systems, multiagent systems, wireless networking and applications in smart city and smart factory, and underwater sensor networks. He has authored and/or coauthored 4 research monographs, more than 180 papers in IEEE Transactions and other peer-reviewed journals, and numerous conference papers. As a Principal Investigator, he has finished/been working on many national key projects. $\mathrm{He}$ is the leader of the prestigious Innovative Research Team awarded by National Natural Science Foundation of China (NSFC). Dr. Guan is an Executive Committee Member of Chinese Automation Association Council and the Chinese Artificial Intelligence Association Council. He was on the editorial board of IEEE Trans. System, Man and Cybernetics?Part C and several Chinese journals. He received the First Prize of Natural Science Award from the Ministry of Education of China in 2006 and the Second Prize of the National Natural Science Award of China in 2008. He was a recipient of the "IEEE Transactions on Fuzzy Systems Outstanding Paper Award" in 2008. He is a "National Outstanding Youth" honored by NSFC, "Changjiang Scholar" by the Ministry of Education of China and "State-level Scholar" of "New Century Bai Qianwan Talent Program" of China. 


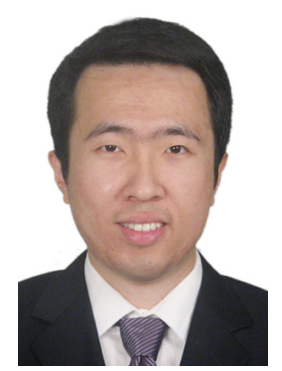

Fan Wu is an associate professor in the Department of Computer Science and Engineering, Shanghai Jiao Tong University. He received his B.S. in Computer Science from Nanjing University in 2004, and $\mathrm{Ph} . \mathrm{D}$. in Computer Science and Engineering from the State University of New York at Buffalo in 2009. He has visited the University of Illinois at Urbana-Champaign (UIUC) as a Post Doc Research Associate. His research interests include wireless networking and mobile computing, algorithmic network economics, and privacy preservation. $\mathrm{He}$ has published more than 70 peer-reviewed papers in leading technical journals and conference proceedings. He is a receipt of China National Natural Science Fund for Outstanding Young Scientists, CCF-Intel Young Faculty Researcher Program Award, CCF-Tencent "Rhinoceros bird" Open Fund, and Pujiang Scholar. He has served as the chair of CCF YOCSEF Shanghai, on the editorial board of Elsevier Computer Communications, and as the member of technical program committees of more than 40 academic conferences. For more information, please visit http://www.cs.sjtu.edu.cn/ fwu/. 